\title{
Control, electronic circuit application and fractional-order analysis of hidden chaotic attractors in the self-exciting homopolar disc dynamo
}

\author{
Zhouchao Wei ${ }^{a, b, c^{*}}$, Akif $\mathrm{Akgul}^{d}$, Uğur Erkin Kocamaz ${ }^{e}$, Irene Moroz ${ }^{c}$, \\ Wei Zhang $f$ \\ ${ }^{a}$ School of Mathematics and Physics, China University of Geosciences, Wuhan, 430074, China \\ ${ }^{b}$ Guangxi Colleges and Universities Key Laboratory of Complex System Optimization and Big Data \\ Processing, Yulin Normal University, Yulin, 537000, China \\ ${ }^{c}$ Mathematical Institute, University of Oxford, Oxford, OX2 6GG, England \\ ${ }^{d}$ Department of Electrical and Electronic Engineering, Faculty of Technology, Sakarya University, \\ Sakarya, 54187, Turkey \\ ${ }^{e}$ Department of Computer Technologies, Vocational School of Karacabey, Uludă̆ University, \\ Karacabey, Bursa, 16700, Turkey \\ ${ }^{f}$ College of Mechanical Engineering, Beijing University of Technology, Beijing, 100124, P. R. China
}

*corresponding author,weizhouchao@163.com

\begin{abstract}
Based on the segmented disc dynamo proposed by H. K. Moffatt, we give out the hidden chaotic attractors, which can show the imperfection in the dynamo model. In this paper. control of hidden chaos in the model is investigated by Lyapunov based nonlinear feedback controllers, sliding mode controllers and hybrid combination of them. Numerical simulations on the comparative analyses are presented. Moreover, with the aid of ORCAD-Pspice and oscilloscope, hidden chaos can be implemented by electronic circuit. Compared with the phase portraits using MATLAB, the simulation results of the oscilloscope outputs verify the effectiveness of electronic circuit design. Finally, in order to consider the effects of fractional order, we analyze the fractional order chaotic system (FOCS) and consider its FPGA implementation for the self-exciting homopolar disc dynamo. The results are helpful for us to better understand the dynamics behavior of disc dynamos.
\end{abstract}

Keywords: Homopolar disc dynamo; Hidden attractor; Chaos control; Circuit application; Fractional order chaotic system; FPGA realization.

\section{Introduction}

The research of chaotic systems is an important and yet difficult task in the disciplines of nonlinear dynamics. The dynamical mechanism of chaotic attractors with unstable equilibria can be applied to designing complex hyperchaotic attractors and communication encryption (Rosa et al., 2000). From another perspective, Hidden periodic states arose in the analysis of 
Hilbert's 16th problem on the number and location of limit cycles in systems with quadratic nonlinearities (Hilbert, 1901). Hidden oscillations can be obtained as a natural feature in electromechanical rotational models and electrical circuits (Sommerfeld, 1902; Blekhman et al., 2007; Eckert, 2013).

Recently, In order to consider dynamical systems with polynomial nonlinearities, oscillations and general issues of stability, etc., the idea of a "hidden attractor" was found in the mid 20th century (Leonov and Kuznetsov, 2013). Hidden chaotic attractors or hyperchaotic attractors have stimulated a lot of research interest (Jafari et al., 2013; Kiseleva et al., 2016; Kuznetsov et al., 2015; Kuznetsov et al., 2016; Wei, 2011; Wei et al., 2016; Wei et al., 2015; Wei and Yang, 2011; Wei and Yang, 2012; Wei et al., 2015). In comparison, hidden chaotic attractors from nonlinear systems with a stable equilibrium, or a nonlinear system without equilibria, are not easy to be localized because the attraction basin for the hidden attractor does not intersect with any small neighborhoods of any equilibria (Leonov and Kuznetsov, 2013; Leonov et al., 2015a; Leonov et al., 2015b; Leonov et al., 2012).

It was traditionally believed that chaotic motions were uncontrollable due to their extreme sensitivity. Researchers frequently encounter chaos control in chaotic systems with engineering background due to the sensitivity of chaos to initial values. The purpose of control is to suppress chaotic motions, and drive them towards one of the system's stable steady states. Hubler (Hubler, 1989) showed that control of chaotic systems can be achieved. Then, Ott et al. (Ott et al., 1990) introduced a chaos control method named OGY. Since these pioneering studies, many researchers have paid attention to control of chaos, such as linear feedback control (Yassen, 2005), nonlinear control (Nijmeijer and Berghuis, 1995; Fang et al., 2000; Park, 2005; Chen, 2005; Pehlivan and Wei, 2012; Aghababa, 2014; Aziz and Al-Azzawi, 2016), adaptive control (Vaidyanathan, 2012), sliding mode control (Yau et al., 2000; Jang et al., 2000; Yau and Yan, 2004; Chang et al., 2008; Ablay, 2009; Motallebzadeh et al.,2009; Kocamaz et al., 2009; Vaidyanathan, 2015), active nonlinear control (Li et al.,2009; Vaidyanathan and Rasappan, 2011), passive control (Chen and Liu, 2010) and backstepping design (Peng and Chen, 2008).

As mentioned in the work by Plunian (Plunian et al., 1998), dynamos will be usefull to understand magnetic field generation and reversals in astrophysical bodies. The Bullard model (Bullard, 1955), known mainly for its educational interest, presents the typical features of a fluid dynamo. The conventional description of the one-disc dynamo has been shown to be misleading from the fundamental point of view. H. K. Moffatt in 1979 (Moffatt, 1979) proposed a self-exciting homopolar dynamo, which segmented the disc by insulating foils, in 
such a way that the current is forced to flow radially, except in the neighbourhood of the disc's rim, where it is azimuthal. The dynamo has not yet been completely well understood because of the existence of hidden chaotic attractors, which is one of important features for imperfect systems. Because hidden chaos is belong to one of the imperfect uncertain systems (Fortuna et al., 2001; Fortuna et al., 2017), the goal of control engineering is to take suitable feedback actions despite imperfections and uncertainties in the model for disc dynamo.

Our objective is to investigate control, electronic circuit application and fractional-order analysis self-exciting homopolar disc dynamo in the paper. Well-known nonlinear based controllers and sliding mode controllers are assigned for controlling the hidden chaos. In order to view the hidden chaos in real time, we design an electronic circuit, and use an oscilloscope in the re-scaled dynamo. Finally, fractional order self-exciting homopolar disc dynamo and its FPGA implementation for the self-exciting homopolar disc dynamo are analyzed.

\section{Hidden attractors in self-exciting homopolar disc dynamo}

\subsection{System Description}

Dynamo models have been established to discuss the generation of magnetic fields and their reversals in astrophysics. Moffatt (Moffatt, 1979) extended the simplest self-exciting Bullard dynamo to include radial diffusion of magnetic field, and obtained the segmented disc dynamo model, which was written non-dimensionally as:

$$
\left\{\begin{array}{l}
\dot{x}=r(y-x) \\
\dot{y}=m x-(1+m) y+x z \\
\dot{z}=g\left[1+m x^{2}-(1+m) x y\right] .
\end{array}\right.
$$

Here $x(t)$ and $y(t)$ denote the magnetic radial and azimuthal current distributions, $z(t)$ is the angular velocity of the disc, and the dot denotes differentiation with respect to time; $g$ measures the applied torque, and $r$ and $m$ are positive constants that depend on the electrical properties of the circuit.

From the following equations

$$
\left\{\begin{array}{l}
r(y-x)=0, \\
m x-(1+m) y+x z=0, \\
g\left[1+m x^{2}-(1+m) x y\right]=0,
\end{array}\right.
$$


we can obtain two equilibria: $E_{1}(-1,-1,1)$ and $E_{2}(1,1,1)$. Linearizing the system (1) about $E_{1,2}$ yields the following characteristic equation:

$$
2 g r+(g+g m) \lambda+(1+m+r) \lambda^{2}+\lambda^{3}=0 .
$$

Base on the Routh-Hurwitz criterion, $E_{1,2}$ are both locally asymptotically stable if and only if

$$
m \geq 1 \text { or } m<1, r<\frac{(m+1)^{2}}{1-m} .
$$

In order to consider hidden chaos in the self-exciting homopolar disc dynamo (1), we firstly give out the comment from Prof. Moffatt: "When $r<\frac{(m+1)^{2}}{1-m}$, it seems probable that all trajectories tend to one of the two equilibrium points" (Moffatt, 1979). However, as we know, multistability depends on the choice of initial conditions, as well as small changes in parameters, so that a sudden transition can occur to a different attractor. Hidden chaos, called one kind of imperfection, can actually exploited to reveal complex dynamics.

\subsection{Hidden chaos}

We now address multistability for Moffatt' inequalities:

$$
\left\{(m, r) \mid m<1, r<\frac{(m+1)^{2}}{1-m}\right\} .
$$

When $r=10, m=0.75, g=20$, we can obtain chaotic attractor, which comes from the effects of different choice of initial data.

(A) For initial values of $(2,2,0.75)$, we obtain a hidden chaotic attractor with Lyapunov exponents: $L_{1}=0.5145, L_{2}=0.0000, L_{3}=-12.2685$. It shows the coexistence of chaos with stable steady states;

(B) When we choose initial conditions $(1.5,1.5,0.75)$, chaotic attractors can not be obtained and Lyapunov exponents: $L_{1}=-0.0332, L_{2}=-0.0332, L_{3}=-11.6836$.

The above Lyapunov exponents are calculated using Wolf's method to run the orbit for a time of $4 \mathrm{e} 7$ using a fourth-order Runge-Kutta integrator with an adaptive step size. They can be obtained from a standard Gram-Schmidt reorthonormalization procedure, using a Jacobian matrix (Wolf et al., 1985; Leonov and Kuznetsov, 2007; Govorukhin, 2004). Note that the behavior of system (1) is very sensitive to initial values. A change in the initial values for $x$ and $y$ lead to very different dynamics. Fig. 1 shows these two coexisting attractors, which show orbit of system (1) will eventually evolve into either a stable steady state or a chaotic 
attractor. This suggests there are still discoveries to be made towards a unified theory for chaotic systems (Wei et al., 2017). In this following sections, we mainly focus on the control, electronic circuit application and fractional-order analysis for the self-exciting homopolar disc dynamo (1) .

\section{Control for the self-exciting homopolar disc dynamo (1)}

In order to drive the chaos of system (1) towards its equilibria control signals $u_{1}, u_{2}$ and $u_{3}$ are added to the $x, y$ and $z$ state variables of the system, respectively:

$$
\left\{\begin{array}{l}
\dot{x}=r(y-x)+u_{1} \\
\dot{y}=m x-(1+m) y+x z+u_{2} \\
\dot{z}=g\left[1+m x^{2}-(1+m) x y\right]+u_{3}
\end{array}\right.
$$

We represent an equilibrium as $\left(x_{d}, y_{d}, z_{d}\right)$, and define perturbations about this state as error states: $e_{1}=x-x_{d}, e_{2}=y-y_{d}$ and $e_{3}=z-z_{d}$. Thus, the equations for the errors $\left(e_{1}, e_{2}, e_{3}\right)$ become:

$$
\left\{\begin{array}{l}
\dot{e}_{1}=r\left(e_{2}-e_{1}+y_{d}-x_{d}\right)+u_{1}, \\
\dot{e}_{2}=\left(m+z_{d}\right) e_{1}-(1+m) e_{2}+x_{d} e_{3}+e_{1} e_{3}+m x_{d}-(1+m) y_{d}+x_{d} z_{d}+u_{2}, \\
\dot{e}_{3}=g\left[1+m\left(e_{1}^{2}+2 x_{d} e_{1}+x_{d}^{2}\right)-(1+m)\left(y_{d} e_{1}+x_{d} e_{2}+e_{1} e_{2}+x_{d} y_{d}\right)\right]+u_{3} .
\end{array}\right.
$$

Since $y_{d}-x_{d}=0, m x_{d}-(1+m) y_{d}+x_{d} z_{d}=0$ and $1+m x_{d}^{2}-(1+m) x_{d} y_{d}=0$, the system

(7) can be simplified as

$$
\left\{\begin{array}{l}
\dot{e}_{1}=r\left(e_{2}-e_{1}\right)+u_{1}, \\
\dot{e}_{2}=\left(m+z_{d}\right) e_{1}-(1+m) e_{2}+x_{d} e_{3}+e_{1} e_{3}+u_{2}, \\
\dot{e}_{3}=g\left[m\left(e_{1}^{2}+2 x_{d} e_{1}\right)-(1+m)\left(y_{d} e_{1}+x_{d} e_{2}+e_{1} e_{2}\right)\right]+u_{3} .
\end{array}\right.
$$

Now we use different control methods to produce asymptotically stable states of system (8) at the zero point.

\subsection{Nonlinear Feedback Control}

We introduce the Lyapunov function

$$
V\left(e_{1}, e_{2}, e_{3}\right)=\frac{1}{2}\left(e_{1}^{2}+e_{2}^{2}+e_{3}^{2}\right)
$$

for system (8), which is positive definite. Differentiating Eq. (9) with respect to time, one has: 


$$
\begin{aligned}
\dot{V}= & e_{1} \dot{e}_{1}+e_{2} \dot{e}_{2}+e_{3} \dot{e}_{3} \\
= & e_{1}\left(r\left(e_{2}-e_{1}\right)\right)+e_{1} u_{1}+e_{2}\left(\left(m+z_{d}\right) e_{1}-(1+m) e_{2}+x_{d} e_{3}+e_{1} e_{3}\right)+e_{2} u_{2} \\
& +e_{3}\left[g\left[m\left(e_{1}^{2}+2 x_{d} e_{1}\right)-(1+m)\left(y_{d} e_{1}+x_{d} e_{2}+e_{1} e_{2}\right)\right]\right]+e_{3} u_{3} .
\end{aligned}
$$

There are many alternatives for the control signals in equation (10). Therefore, we set:

$$
\left\{\begin{array}{l}
u_{1}=0, \\
u_{2}=-\left(m+r+z_{d}\right) e_{1}+m e_{2}-x_{d} e_{3}-e_{1} e_{3}, \\
u_{3}=-g\left[m\left(e_{1}^{2}+2 x_{d} e_{1}\right)-(1+m)\left(y_{d} e_{1}+x_{d} e_{2}+e_{1} e_{2}\right)\right]-e_{3} .
\end{array}\right.
$$

Then, equation (10) becomes

$$
\dot{V}=-r e_{1}^{2}-e_{2}^{2}-e_{3}^{2},
$$

which vanishes at the equilibrium of system (8) and is negative definite as $r>0$. Consequently, from Lyapunov's direct stability method, error system (8) will converge to zero asymptotically with the choice of controllers in Eq. (11). Hence, the control of the self-exciting homopolar disc dynamo (6) is completed.

\subsection{Sliding Mode Control}

System (8) can be written as

$$
\dot{e}=A e+\eta(x, y, z)+u,
$$

where

$$
\begin{aligned}
& A=\left[\begin{array}{ccc}
-r & r & 0 \\
m+z_{d} & -(1+m) & x_{d} \\
g\left[2 m x_{d}-(1+m) y_{d}\right] & -g\left[(1+m) x_{d}\right] & 0
\end{array}\right], \\
& \eta(x, y, z)=\left[\begin{array}{c}
0 \\
-e_{1} e_{3} \\
g\left[m e_{1}^{2}-(1+m) e_{1} e_{2}\right.
\end{array}\right],
\end{aligned}
$$

and $u=\left[u_{1} u_{2} u_{3}\right]^{T}$. Basing on sliding mode control theory, the formula of the control signal $u$ is:

$$
u=-\eta(x, y, z)+B v,
$$

where $B$ is a column vector and $v$ is a control function (Vaidyanathan, 2015). For the purpose of control, the matrix

$$
\left[I-B(C B)^{-1} C\right] A
$$


must have all its eigenvalues in the left half plane. Here, $I$ is the identity matrix, $C$ is a row vector and $B=\left[\begin{array}{lll}0 & 1 & 1\end{array}\right]^{\mathrm{T}}$ for ensuring that $(A, B)$ is controllable (Vaidyanathan, 2015). It is easy to know the controlled system will be globally stable for an appropriate choice $C$ vector. To make sliding mode control simpler to be completed, we take $C=\left[\begin{array}{ll}1-5 x_{d} & 4\end{array}\right]$. Then, the sliding surface $s$ is given by

$$
s=C e=\left[\begin{array}{lll}
1 & -5 x_{d} & 4
\end{array}\right] e=e_{1}-5 x_{d} e_{2}+4 e_{3},
$$

and the control function $v$ is:

$$
v=-(C B)^{-1}[C(k I+A) e+q \operatorname{sign}(s)]
$$

where $k$ and $q$ are positive real control gains. Large values of $k$ lead to chattering but will decrease the time taken to approach the sliding surface; small values of $q$ reduce chattering but will increase this time of approach.

The control function $v$ is acquired from Eq. (19) as

$$
v=-\frac{1}{4-5 x_{d}}\left[\begin{array}{l}
\left(k-r-5\left(m+z_{d}\right) x_{d}+4 g\left(2 m x_{d}-(1+m) y_{d}\right)\right) e_{1} \\
+\left(r-5(k-1-m) x_{d}-4 g(1+m) x_{d}\right) e_{2}+\left(4 k-5 x_{d}^{2}\right) e_{3}+q \operatorname{sign}(s)
\end{array}\right] .
$$

Eventually, from Eq. (16), the required sliding mode control signals become

$$
\left\{\begin{array}{l}
u_{1}=0 \\
u_{2}=e_{1} e_{3}+v \\
u_{3}=-g\left[m e_{1}^{2}-(1+m) e_{1} e_{2}\right]+v .
\end{array}\right.
$$

We have therefore completed the control of the disc dynamo (6) with hidden chaos with the sliding mode control signals via Eqs. (18), (20) and (21).

\subsection{Hybrid Control}

We now combine the nonlinear feedback control and sliding mode control methods in a novel control strategy. The error system (8) is again considered. There are now two control functions, so there are two alternative solutions, which we now describe.

\section{Case A.}

If the nonlinear controller $u_{2}$ is taken as

$$
u_{2}=-\left(m+r+z_{d}\right) e_{1}+m e_{2}-x_{d} e_{3}-e_{1} e_{3}+g(1+m)\left(x_{d} e_{3}+e_{1} e_{3}\right),
$$

we obtain the error equations: 


$$
\left\{\begin{array}{l}
\dot{e}_{1}=r\left(e_{2}-e_{1}\right)+u_{1}, \\
\dot{e}_{2}=-r e_{1}-e_{2}+g(1+m)\left(x_{d} e_{3}+e_{1} e_{3}\right), \\
\dot{e}_{3}=g\left[m\left(e_{1}^{2}+2 x_{d} e_{1}\right)-(1+m)\left(y_{d} e_{1}+x_{d} e_{2}+e_{1} e_{2}\right)\right]+u_{3} .
\end{array}\right.
$$

Differentiating the Lyapunov function $V$ with respect to time gives

$$
\begin{aligned}
\dot{V} & =e_{1} \dot{e}_{1}+e_{2} \dot{e}_{2}+e_{3} \dot{e}_{3} \\
& =-r e_{1}^{2}+e_{1} u_{1}-e_{2}^{2}+g e_{1} e_{3}\left[m\left(e_{1}+2 x_{d}-y_{d}\right)-y_{d}\right]+e_{3} u_{3} .
\end{aligned}
$$

From Eq. (24), we can see that $e_{2}$ is either zero or negative definite. Therefore, $e_{2}$ will converge to zero as $t$ tends infinity. (23) can be simplified to

$$
\left\{\begin{array}{l}
\dot{e}_{1}=-r e_{1}+u_{1}, \\
\dot{e}_{3}=g e_{1}\left[m\left(e_{1}+2 x_{d}-y_{d}\right)-y_{d}\right]+u_{3} .
\end{array}\right.
$$

Next we apply the sliding mode control and Eq. (25) can be expressed in matrix form:

$$
\dot{e}=\left[\begin{array}{c}
\dot{e}_{1} \\
\dot{e}_{3}
\end{array}\right]=\underbrace{\left[\begin{array}{cc}
-r & 0 \\
g\left(m\left(2 x_{d}-y_{d}\right)-y_{d}\right) & 0
\end{array}\right]}_{A} \underbrace{\left[\begin{array}{c}
e_{1} \\
e_{3}
\end{array}\right]}_{e}+\underbrace{\left[\begin{array}{c}
0 \\
g m e_{1}^{2}
\end{array}\right]}_{\eta(x, y, z)}+\underbrace{\left[\begin{array}{c}
u_{1} \\
u_{3}
\end{array}\right]}_{u} .
$$

$B$ is taken as $B=\left[\begin{array}{ll}0 & 1\end{array}\right]^{\mathrm{T}}$. The sliding mode theory leads to the sliding surface

$$
s=C e=\left[\begin{array}{ll}
-1 & 5
\end{array}\right] e=-e_{1}+5 e_{3} .
$$

The control function $v$ is

$$
v=-\frac{1}{5}\left[\left(-k+r+5 g\left(m\left(2 x_{d}-y_{d}\right)-y_{d}\right)\right) e_{1}+5 k e_{3}+q \operatorname{sign}(s)\right] .
$$

Afterward, the required sliding mode control signals become

$$
\left\{\begin{array}{l}
u_{1}=0 \\
u_{3}=-g m e_{1}^{2}+v .
\end{array}\right.
$$

The controlled system (6) is thereby completed with a hybrid combination of nonlinear and sliding mode control signals via Eqs. (22), (28) and (29).

\section{Case B.}

If the nonlinear controller $u_{3}$ is taken as

$$
u_{3}=-g\left[m\left(e_{1}^{2}+2 x_{d} e_{1}\right)-(1+m)\left(y_{d} e_{1}+x_{d} e_{2}+e_{1} e_{2}\right)\right]-x_{d} e_{2}-e_{1} e_{2}-e_{3},
$$

the errors satisfy

$$
\left\{\begin{array}{l}
\dot{e}_{1}=r\left(e_{2}-e_{1}\right)+u_{1}, \\
\dot{e}_{2}=\left(m+z_{d}\right) e_{1}-(1+m) e_{2}+x_{d} e_{3}+e_{1} e_{3}+u_{2}, \\
\dot{e}_{3}=-x_{d} e_{2}-e_{1} e_{2}-e_{3} .
\end{array}\right.
$$


Again differentiating $V$ with respect to time gives

$$
\begin{aligned}
\dot{V} & =e_{1} \dot{e}_{1}+e_{2} \dot{e}_{2}+e_{3} \dot{e}_{3} \\
& =e_{1}\left(r\left(e_{2}-e_{1}\right)\right)+e_{1} u_{1}+e_{2}\left(\left(m+z_{d}\right) e_{1}-(1+m) e_{2}\right)+e_{2} u_{2}-e_{3}^{2} .
\end{aligned}
$$

Eq. (32) shows that now $e_{3}$ is zero or negative definite. Therefore, $e_{3}$ will converge to zero as $t$ tends infinity. We obtain the error evolution equations:

$$
\left\{\begin{array}{l}
\dot{e}_{1}=r\left(e_{2}-e_{1}\right)+u_{1}, \\
\dot{e}_{2}=\left(m+z_{d}\right) e_{1}-(1+m) e_{2}+u_{2} .
\end{array}\right.
$$

Next we apply the sliding mode control, and re-write Eq. (33) in matrix form:

$$
\dot{e}=\left[\begin{array}{l}
\dot{e}_{1} \\
\dot{e}_{2}
\end{array}\right]=\underbrace{\left[\begin{array}{cc}
-r & r \\
m+z_{d} & -(1+m)
\end{array}\right]}_{A} \underbrace{\left[\begin{array}{l}
e_{1} \\
e_{2}
\end{array}\right]}_{e}+\underbrace{\left[\begin{array}{c}
0 \\
0
\end{array}\right]}_{\eta(x, y, z)}+\underbrace{\left[\begin{array}{l}
u_{1} \\
u_{2}
\end{array}\right]}_{u} .
$$

$B$ is taken as $B=\left[\begin{array}{ll}0 & 1\end{array}\right]^{\mathrm{T}}$. The sliding surface is

$$
s=C e=\left[\begin{array}{ll}
4 & 5
\end{array}\right] e=4 e_{1}+5 e_{2},
$$

while the control function $v$ is

$$
v=-\frac{1}{5}\left[\left(4(k-r)+5\left(m+z_{d}\right)\right) e_{1}+(4 r+5(k-1-m)) e_{2}+q \operatorname{sign}(s)\right]
$$

Afterward, the required sliding mode control signals become

$$
\left\{\begin{array}{l}
u_{1}=0 \\
u_{2}=v
\end{array}\right.
$$

This completes the analysis of the hybrid controlled system (6) with Eqs. (30), (36) and (37).

\subsection{Numerical Simulations}

As shown in Fig. 1, for $r=10, m=0.75, g=20$, system (1) has hidden chaos. To demonstrate the control of chaotic behavior in the dynamo, we used a fourth-order Runge-Kutta scheme, and take a fixed time step of 0.0001 in numerical integrations. The gains of the controllers are selected as $k_{1}=1, k_{2}=1, k=1$ and $q=0.1$.

When the controllers were activated at $t=15$ and $t=25$, nonlinear feedback controllers, sliding mode controllers and hybrid controllers are deployed for the control with initial conditions $(0.2,0.2,2)$. Simulations show evolution towards equilibrium $E_{1}(-1,-1,1)$, as shown in Figs. 2 and 3, respectively. For equilibrium $E_{2}(1,1,1)$, the results are shown in Figs. 4 and 5, respectively.

After activating the controllers, successful control of the system is seen for all signals and methods, thereby, validating the theoretical analyses. In the simulations, the signal $z$ generally 
designates the effectiveness of overall control. When the control is activated, it is observed with nonlinear feedback controllers around 5-6 times the period, sliding mode controllers around 4-5 times the period, while the hybrid controllers in Case B at around 3-4 times period, and the control is activated at twice the period with the hybrid controllers in Case A . Therefore, the simulation results show that the proposed hybrid controllers in Case A produce an improved performance in control time.

From the above control methods, we can know each of the nonlinear controllers mentioned in the introduction has unique merits. But it is believed that a hybrid control approach may be able to take advantage of several algorithms' benefits, and thus address the unique properties and needs of the various magnetic bearing subsystems. In addition, the overall control scheme would be divided into smaller subsystems, each of which may be easier to design than a full-state, nonlinear controller for the entire system dynamics.

\section{The electronic circuit implementation of 3D chaotic system (1)}

To prevent electronic components such as operational amplifiers saturating, the variable amplitudes are reduced by linearly re-scaling them (Akgul et al.,2016a; Akgul et al.,2016b; Sundarapandian and Pehlivan, 2012; Pehlivan et al.,2014; Cicek et al.,2016; Kacar, 2016; Cicek et al., 2016; Buscarino et al., 2011). Fig. 1 shows the $x, y$ and $z$ signals in the interval of $(-20,30)$. The values for $x, y$ do not require rescaling because their values fall within the interval of $(-15,15)$. However, $z$ has to be rescaled for real-time observations on the oscilloscope.

Under the transform $X=x, Y=y$ and $Z=z / 2$, the self-exciting homopolar disc dynamo (1) becomes:

$$
\left\{\begin{array}{l}
\dot{X}=r X-2 r Z \\
\dot{Y}=m X-(1+m) Y+2 X Z \\
\dot{Z}=\frac{g\left[1+m X^{2}-(1+m) X Y\right]}{2} .
\end{array}\right.
$$

By using ORCAD-Pspice to design and implementing the electronic circuit on the oscilloscope, we can obtain the schematic in Fig. 6, where the circuit comprises standard electronic components such as resistors, capacitors, op-amps and multipliers.

We continue to choose the parameter values for the hidden chaos: $r=10, m=0.75, g=20$ and initial conditions $x(0)=0.3, y(0)=2.9, z(0)=0.1$. TL081 op-amps and Analog Device AD633 multipliers are used, as well as resistors with $\mathrm{R}_{1}=\mathrm{R}_{2}=40 \mathrm{Kohm}, \mathrm{R}_{3}=533.33 \mathrm{Kohm}$, 
$\mathrm{R}_{4}=288.5 \mathrm{Kohm}, \mathrm{R}_{5}=20 \mathrm{Kohm}, \mathrm{R}_{6}=600 \mathrm{Kohm}, \mathrm{R}_{7}=5.33 \mathrm{Kohm}, \mathrm{R}_{8}=2.28 \mathrm{Kohm}, \mathrm{R}_{9}=$ $\mathrm{R}_{10}=\mathrm{R}_{11}=\mathrm{R}_{12}=100 \mathrm{Kohm}$, and capacitors $\mathrm{C}_{1}=\mathrm{C}_{2}=\mathrm{C}_{3}=1 \mathrm{nF}, \mathrm{V}_{\mathrm{n}}=-15 \mathrm{~V}, \mathrm{~V}_{\mathrm{p}}=15 \mathrm{~V}$ are chosen. Inputs to the AD633 multiplier IC are constrained to lie between $-10 \mathrm{~V}$ and $+10 \mathrm{~V}$. The output voltage is the product of the inputs divided by $10 \mathrm{~V}$. Real-time realizations of the chaos were made using the electronic card shown in Fig. 7. The oscilloscope outputs of the scaled system are shown in Figs.8, which shows consistent with corresponding plots from MATLAB integrations in Fig.1. Therefore, it is effective for the electronic circuit design and experiments.

\section{Analysis of a fractional order chaotic system (FOCS) for the self-exciting homopolar disc dynamo (1)}

Self-exciting homopolar disc dynamo is one more dynamic system, which play significant role to describe the generation of magnetic fields. With the application of fractional-order systems, we will consider chaos in the homopolar disc dynamo of total order less than 3 in this section.

Based on definition of the fractional-order differential operator, viz. Grunwald-Letnikov, Riemann-Liouville and Caputo (Cicek et al.,2016; Zhou, 2014; Diethelm, 2010), we will study the dynamical behavior of fractional order model of self-exciting homopolar disc dynamo (1) by the Grunwald -Letnikov (GL) definition

$$
D_{t}^{q} f(t)=\lim _{h \rightarrow 0}\left\{\frac{1}{h^{q}} \sum_{j=0}^{\left[\frac{t-q}{h}\right]}(-1)^{j}\left(\begin{array}{c}
q \\
j
\end{array}\right) f(t-j h)\right\}=\lim _{h \rightarrow 0}\left\{\frac{1}{h^{q}} \Delta_{h}^{q} f(t)\right\},
$$

where $a$ and $t$ are limits of the fractional order system, $\Delta_{h}^{q} f(t)$ is generalized difference, $h$ is step size and $q$ is the fractional order.

The above equation (39) can be further modified as

$$
{ }_{(t-L)} D_{t}^{q} f(t)=\lim _{h \rightarrow 0}\left\{h^{-q} \sum_{j=0}^{N(t)} b_{j}(f(t-j h))\right\},
$$

where the binomial coefficient

$$
b_{j}=\left(1-\frac{a+q}{j}\right) b_{j-1} .
$$

In order to calculate the fractional order system, we can use finite memory principal, where $L$ is the memory length and $h$ is the time sampling. 


$$
N(t)=\min \left\{\left[\frac{t}{h}\right],\left[\frac{L}{h}\right]\right\}
$$

Therefore, the fractional order 3D chaotic system is derived as described:

$$
\begin{aligned}
& D^{q_{x}} x=r(y-x), \\
& D^{q_{y}} y=m x-(1+m) y+x z, \\
& D^{q_{z}} z=g\left[1+m x^{2}-(1+m) x y\right],
\end{aligned}
$$

where $r=10, m=0.75, g=20$ and commensurate fractional order $q=0.997$. The 2D phase portraits of the FOCS system is shown in Fig. 9.

\subsection{Dynamic analysis of the FOCS system (43)}

Most of the dynamic properties of the FOCS systems like the Lyapunov exponents and bifurcation with parameters are preserved in the FOCS system (Rajagopal et al.,2017d; Rajagopal et al.,2017c) if $q_{i}>0.994$ where $i=x, y, z$. In Fig.10, the bifurcation of the FOCS system with parameter $r$ for backward continuation can be obtained in red points. The red points of bifurcation show that the FOCS system has multiple chaotic regions for parameter $r$. The FOCS shows the highest Lyapunov exponent in the region $9 \leq r \leq 11.8$. Two small quasi chaotic regions can be seen for $11.8 \leq r \leq 11.83$ and $12.2 \leq r \leq 12.3$, where the only positive Lyapunov exponent becomes zero. The third chaotic region exists for $12.3 \leq r \leq 13.5$. The FOCS system exists the chaotic region by inverse period doubling bifurcations. The fourth chaotic region exists for the range $14.4 \leq r \leq 15.2$ and the FOCS system exits the chaotic region via a second inverse period doubling.

Furthermore, we made some adjustments and tried forward continuation with initial conditions $(2,2,0.75)$ and found coexisting attractors. When $r$ in $(9,13)$, it is easy to see the multistability and coexisting attractors in the fractional order system (blue part means stable equilibrium, red part means chaos), shown in blue points in Fig.10. Fig.11 shows the bifurcation of the FOCS chaotic systems for variation in fractional orders $q_{x}=q_{y}=q_{z}=q$. The largest positive Lyapunov exponent $\left(\mathrm{L}_{1}=0.5516\right)$ of the FOCS appears when $q=0.997$. When the fractional order $q$ decreases, the FOCS systems starts losing its largest positive Lyapunov exponent. When $q \leq 0.994$, the chaotic behavior will not exist as the only positive Lyapunov exponent of the system turns to a negative value. 
Remark: It is found that the decreasing of the self-exciting homopolar disc dynamo model's order has great effect on the dynamics of this nonlinear system. the change of fractional order has little effect on the bandwidth of frequency spectrum using the time domain approximation method. According to the bifurcation analysis, the fractional order disc dynamo system can show period bifurcation and chaotic attractors.

\subsection{FPGA implementation of the FOCS system (43)}

From subsection 5.1, fractional order self-exciting homopolar disc dynamo also present hidden attractors depend on the parameters and order. Therefore, we consider to realize the imperfection in real world applications in this section.

Let fractional order $q=0.997$ in system (43), we will discuss the implementation in FPGA using the Xilinx (Vivado) System Generator (Rajagopal et al.,2017a; Rajagopal et al.,2017b; Rajagopal et al., 2017d; Rajagopal et al., 2017c; Tlelo-Cuautle et al., 2016; Wang et al., 2016; Dong et al., 2016; Tlelo-Cuautle et al., 2015a; Rashtchi and Nourazar, 2015; Tlelo-Cuautle et al., 2015b; Xu et al., 2016). Here we implement the integrators using the mathematical relation (Chen et al., 2004) and the value of time sampling interval $h$ is taken as 0.001 seconds in (42). The initial conditions are fed in to the forward register. As we know, the results on FPGA is directly related to the number of threads and its performances, therefore FOCS system (43) with fractional order taken as $q=0.997$ will be designed as four parallel threads (Rajagopal et al.,2017d). Fig. 12 shows the RTL schematics of the FOCS system. Fig.13a and 13b shows the power utilization and power consumption for various fractional orders, table I shows the resource consumption. Fig 14 shows the 2D phase portraits of the FOCS system.

\section{Conclusion}

In this paper, we investigated the control of dynamics in a simplified three-dimensional self-exciting homopolar disc dynamo, which can exhibit hidden chaos and coexisting attractors without unstable equilibria. The control of chaotic system with only stable equilibria can be achieved by means of nonlinear controllers, sliding mode controllers and a hybrid combination of them. Based on the Lyapunov stability and sliding mode control theories, the conditions for asymptotic stability of the steady states are provided theoretically. An electronic circuit of the rescaled system is implemented using the ORCAD-PSpice program. Numerical simulations validated the theoretical analyses. A fractional order model 
of the self-exciting homopolar disc dynamo is derived and implemented in FPGA. Power efficiency analysis for various fractional orders are derived and it is shown that the fractional order disc dynamo system can show period bifurcation and chaotic attractors. Such analysis about imperfection for the suitable physical example will form the basis of more systematic studies of hidden chaos in a forthcoming study.

\section{Declaration of Conflicting Interests}

The author(s) declared no potential conflicts of interest with respect to the research, authorship, and/or publication of this article.

\section{Acknowledgements}

We thank the Mathematical Institute, University of Oxford for providing the facilities to enable this research to undertaken. This work was supported by the Natural Science Foundation of China (No.11772306 and No.11401543), Scientific Research Program of Hubei Provincial Department of Education (No.B2017599), the Open Foundation for Guangxi Colleges and Universities Key Lab of Complex System Optimization and Big Data Processing (No.2016CSOBDP0202), the Fundamental Research Funds for the Central Universities, China University of Geosciences (Wuhan) (No. CUGL150419), and Sakarya University Scientific Research Projects Unit (No. 201609-00-008).

\section{References}

Ablay G (2009) Sliding mode control of uncertain unified chaotic systems. Nonlinear Anal.: Hybrid Syst. 3(4): 531-535.

Aghababa MP (2014) A Lyapunov-based control scheme for robust stabilization of fractional chaotic systems. Nonlinear Dyn. 78(3): 2129-2140.

Akgul A, Moroz I, Pehlivan I and Sundarapandian V (2016a) A new four-scroll chaotic attractor and its engineering applications. Optik 127(13): 5491-5499.

Akgul A, Shafqat H and Pehlivan I (2016b) A new three-dimensional chaotic system, its dynamical analysis and electronic circuit applications. Optik 127(18): 7062-7071.

Aziz MM and Al-Azzawi SF (2016) Control and synchronization with known and unknown parameters. Appl. Math. 7: 292-303.

Baleanu D, Diethelm K, Scalas E and Trujillo JJ (2014) Fractional Calculus: Models and Numerical Methods, World Scientific, Singapore.

Blekhman I, Indeitsev DA and Fradkov AL (2007) Slow motions in systems with inertially excited vibrations. IFAC Proceedings 40(14): 126-131.

Bullard EC (1955) The stability of a homopolar dynamo. Proc. Camb. Phil. Soc. 51: 744-760.

Buscarino A., Fortuna L, Frasca M, Sciuto G (2011) Design of time-delay chaotic electronic circuits. IEEE Trans. Circuits Syst. I Regul. Pap. 58: 1888-1896. 
Chang JF, Hung ML, Yang YS, Liao TL and Yan JJ (2008) Controlling chaos of the family of Rössler systems using sliding mode control. Chaos Solitons Fract. 37(2): 609-622.

Chen HK (2005) Global chaos synchronization of new chaotic systems via nonlinear control. Chaos Solitons Fract. 23(4): 1245-1251.

Chen XR and Liu CX (2010) Passive control on a unified chaotic system. Nonlinear Anal.: Real World Appl. 11(2): 683-687.

Chen YQ, Vinagre BM and Podlubny I (2004) Continued fraction expansion approach to discretizing fractional order derivatives-an expository review. Nonlinear Dyn. 38:155-170.

Cicek S, Ferikoglu A and Pehlivan I (2016) A new 3D chaotic system: Dynamical analysis, electronic circuit design, active control synchronization and chaotic masking communication application. Optik 127(8): 4024-4030.

Coskun S, Tuncel S, Pehlivan I and Akgul A (2015) Microcontroller-controlled electronic circuit for fast modelling of chaotic equations. Electronics World 121(1947): 24-25.

Diethelm K (2010) The Analysis of Fractional Differential Equations, Springer, Berlin.

Dong EZ, Liang ZH and Du SZ (2016) Topological horseshoe analysis on a four-wing chaotic attractor and its FPGA implementation. Nonlinear Dyn. 83(1-2): 623-630.

Eckert M (2013) Arnold Sommerfeld: Science, life and turbulent times 1868-1951. New York, Springer-Verlag.

Fang JQ, Hong YG, Qin HS and Chen GR (2000) Nonlinear control of chaotic systems: A switching manifold approach. Discrete Dyn. Nature Society 4(4): 257-267.

Fortuna L, Arena P, Bálya D, Zarándy Á (2001) Cellular neural networks: a paradigm for nonlinear spatio-temporal processing. IEEE Circ. Syst. Mag. 1(4): 6-21.

Fortuna L, Buscarino A, Frasca M, Famoso C (2017) Control of Imperfect Nonlinear Electromechanical Large Scale Systems: From Dynamics to Hardware Implementation. World Scientific Series on Nonlinear Science, Series A - Vol. 50, Singapore.

Govorukhin VN (2004) see http://kvm.math.rsu.ru/matds/ for MATDS.

Hilbert D (1901) Mathematical problems. Bull. Amer. Math. Soc. 8: 437-479.

Hubler A (1989) Adaptive control of chaotic systems. Helvetica Physica Acta 62(2-3): 343-346.

Jafari S, Sprott JC and Golpayegani SMRH (2013) Elementary quadratic chaotic flows with no equilibria. Phys. Lett. A 377(9): 699-702.

Jang MJ, Chen CL, Chen CK (2002) Sliding mode control of chaos in the cubic Chua's circuit system. Int. J. Bifurcat. Chaos 12(6): 1437-1449.

Kacar S (2016) Analog circuit and microcontroller based RNG application of a new easy realizable 4D chaotic system. Optik 127(20): 9551-9561.

Kiseleva MA, Kuznetsov NV and Leonov GA (2016) Hidden attractors in electromechanical systems with and without equilibria. IFAC-PapersOnLine 49(14): 51-55.

Kocamaz UE, Uyaroglu Y and Kizmaz H (2014) Control of Rabinovich chaotic system using sliding mode control. Int. J. Adapt. Control Signal Process. 28(12): 1413-1421

Kuznetsov AP, Kuznetsov SP, Mosekilde E and Stankevich NV (2015) Co-existing hidden attractors in a radio-physical oscillator system. J. Phys. A: Math. Theor. 48(12): 125101.

Kuznetsov NV, Leonov GA, Mokaev TN and Seledzhi SM (2016) Hidden attractor in the Rabinovich system, Chua circuits and PLL. AIP Conf. Proc. 1738: 210008.

Leonov GA and Kuznetsov NV (2007) Time-varying linearization and the Perron effects. Int. J. Bifurcat. Chaos 17: 1079-1107.

Leonov GA and Kuznetsov NV (2013) Hidden attractors in dynamical systems: From hidden oscillations in Hilbert-Kolmogorov, Aizerman and Kalman problems to hidden chaotic attractor in Chua circuits. Int. J. Bifurcat. Chaos 23(1): 1330002. 
Leonov GA, Kuznetsov NV and Mokaev TN (2015a) Homoclinic orbits, and self-excited and hidden attractors in a Lorenz-like system describing convective fluid motion. Eur. Phys. J. Spec. Top. 224(8): 1421-1458.

Leonov GA, Kuznetsov NV and Mokaev TN (2015b) Homoclinic orbit and hidden attractor in the Lorenz-like system describing the fluid convection motion in the rotating cavity. Commun. Nonlinear Sci. Numer. Simul. 28(1-3): 166-174.

Leonov GA, Kuznetsov NV and Vagaitsev VI (2012) Hidden attractor in smooth Chua systems. Physica D 241(18): 1482-1486.

Li Y, Yusheng Z, Hang X, Shaoming W and Wei L (2001) The nonlinear active control of chaos in thermal convection loop. Int. Conf. on Energy Conversion and Application, Wuhan, China, June 17-20, pp. 453-456.

Moffatt HK (1979) A self consistent treatment of simple dynamo systems. Geophys. Astrophys. Fluid Dyn. 14: 147-166.

Motallebzadeh F, Dadras S, Motallebzadeh F and Ozgoli S (2009) Controlling chaos in Arneodo system. 17th Mediterranean Conf. on Control \& Automation, IEEE, Thessaloniki, Greece, June 24-26, pp. 314-319.

Nijmeijer H and Berghuis H (1995) On Lyapunov control of the Duffing equation. IEEE Trans. Circuits-I 42(8): 473-477.

Ott E, Grebogi C and Yorke JA (1990) Controlling chaos. Phys. Rev. Lett. 64(11): 1196-1199.

Park JH (2005) Chaos synchronization of a chaotic system via nonlinear control. Chaos Solitons Fract. 25(3): 579-584.

Pehlivan I, Moroz I and Sundarapandian V (2014) Analysis, synchronization and circuit design of a novel butterfly attractor. J. Sound Vibration 333(20): 5077-5096.

Pehlivan I and Wei ZC (2012) Analysis, nonlinear control, and chaos generator circuit of another strange chaotic system. Turk. J. Electr. Engin. Comput. Sci. 20: 1229-1239.

Peng CC and Chen CL (2008) Robust chaotic control of Lorenz system by backstepping design. Chaos Solitons Fract. 37(2): 598-608.

Plunian F, Marty Ph and Alemany A (1998) Chaotic behaviour of the Rikitake dynamo with symmetric mechanical friction and azimuthal currents. Proc. $R$. Soc. Lond. A 454:1835-1842.

Rajagopal K, Guessas L,Vaidyanathan S, Karthikeyan A and Srinivasan A (2017a) Dynamical Analysis and FPGA Implementation of a Novel Hyperchaotic System and Its Synchronization Using Adaptive Sliding Mode Control and Genetically Optimized PID Control. Math. Probl. Eng. 2017: 7307452

Rajagopal K, Karthikeyan A and Duraisamy P (2017b) Hyperchaotic Chameleon: Fractional order FPGA implementation. Complexity 2017: 8979408.

Rajagopal K, Karthikeyan A and Srinivasan A (2017c) FPGA implementation of novel fractional order chaotic system with two equilibriums and no equilibrium and its adaptive sliding mode synchronization. Nonlinear Dyn. 87 (4): 2281-2304 .

Rajagopal K, Laarem G, Karthikeyan A, Srinivasan A andAdam G (2017d) Fractional order memristor no equilibrium chaotic system with its adaptive sliding mode synchronization and genetically optimized fractional order PID synchronization. Complexity 2017: 1892618.

Rashtchi V and Nourazar M (2015) FPGA Implementation of a Real-Time Weak Signal Detector Using a Duffing Oscillator. 34(10): 3101-3119.

Rosa ML, Rabinovich MI, Huerta R, Abarbanel HDI and Fortuna L (2000) Slow regularization through chaotic oscillation transfer in an unidirectional chain of Hindmarsh-Rose models. Phys. Lett. A 266, 88-93. 
Sommerfeld A (1902) Beitrage zum dynamischen ausbau der festigkeitslehre. Z. Vereins Deutscher Ingenieure 46: 391-394.

Sundarapandian V and Pehlivan I (2012) Analysis, control, synchronization and circuit design of a novel chaotic system. Math. Comput. Model. 55(7-8): 1904-1915.

Tlelo-Cuautle E, Carbajal-Gomez, VH and Obeso-Rodelo PJ (2015a) FPGA realization of a chaotic communication system applied to image processing. Nonlinear Dyn. 82(4): 1879-1892.

Tlelo-Cuautle E, Pano-Azucena AD and Rangel-Magdaleno JJ (2016) Generating a 50-scroll chaotic attractor at $66 \mathrm{MHz}$ by using FPGAs. Nonlinear Dyn. 85(4): 2143-2157 .

Tlelo-Cuautle E, Rangel-Magdaleno and Rangel-Magdaleno JJ (2015b) FPGA realization of multi-scroll chaotic oscillators. Commun. Nonlinear Sci. Numer. Simul. 27(1-3): 66-80.

Vaidyanathan S (2015) Sliding mode control of Rucklidge chaotic system for nonlinear double convection. Int. J. Chem. Tech. Research 8(8): 25-35.

Vaidyanathan S (2012) Adaptive control and synchronization of Shimizu-Morioka chaotic system. Int. J. in Foundations of Computer Science \& Technology 2(4): 29-42.

Vaidyanathan S, Rasappan S (2011) Global chaos synchronization of hyperchaotic Bao and Xu systems by active nonlinear control. Commun. Computer Inform. Sci. 198: 10-17.

Wang QX, Yu SM and Li CQ (2016) Theoretical Design and FPGA-Based Implementation of Higher-Dimensional Digital Chaotic System. IEEE Trans. Circuits-I 63(3): 401-412.

Wei ZC (2011) Dynamical behaviors of a chaotic system with no equilibria. Phys. Lett. A 376(2): 102-108.

Wei ZC, Moroz I, Sprott JC, Wang Z and Zhang W (2017) Detecting hidden chaotic regions and complex dynamics in the self-exciting homopolar disc dynamo. Int. J. Bifurcat. Chaos 27(2): 1730008.

Wei ZC, Moroz I, Wang Z, Sprott JC and Kapitaniak T (2016) Dynamics at infinity, degenerate Hopf and zero-Hopf bifurcation for Kingni-Jafari system with hidden attractors. Int. J. Bifurcat. Chaos 26(7): 1650125.

Wei ZC, Sprott JC and Chen H (2015a) Elementary quadratic chaotic flows with a single non-hyperbolic equilibrium. Phys. Lett. A 379(37): 2184-2187.

Wei ZC and Yang QG (2011) Dynamical analysis of a new autonomous 3-D chaotic system only with stable equilibria. Nonlinear Anal.: Real World Appl. 12(1): 106-118.

Wei ZC and Yang QG (2012) Dynamical analysis of the generalized Sprott C system with only two stable equilibria. Nonlinear Dyn. 68(4): 543-554.

Wei ZC, Zhang W, Wang Z and Yao MH (2015b) Hidden attractors and dynamical behaviors in an extended Rikitake system. Int. J. Bifurcat. Chaos 25(2): 1550028.

Wolf A, Swift JB, . Swinney HL and Vastano JA (1985) Determining Lyapunov exponents from a time series. Physica D 16: 285-317.

Xu YM, Wang LD and Duan K (2016) A memristor-based chaotic system and its field programmable gate array implementation. Acta Physica Sinica. 65(12): 120503.

Yassen MT (2005) Controlling chaos and synchronization for new chaotic system using linear feedback control. Chaos Solitons Fract. 26(3): 913-920.

Yau HT, Chen CK and Chen CL (2000) Sliding mode control of chaotic systems with uncertainties. Int. J. Bifurcat. Chaos 10(5): 1139-1147.

Yau HT and Yan JJ (2004) Design of sliding mode controller for Lorenz chaotic system with nonlinear input. Chaos Solitons Fract. 19(4): 891-898.

Zhou Y (2014) Basic Theory of Fractional Differential Equations, World Scientific, Singapore. 

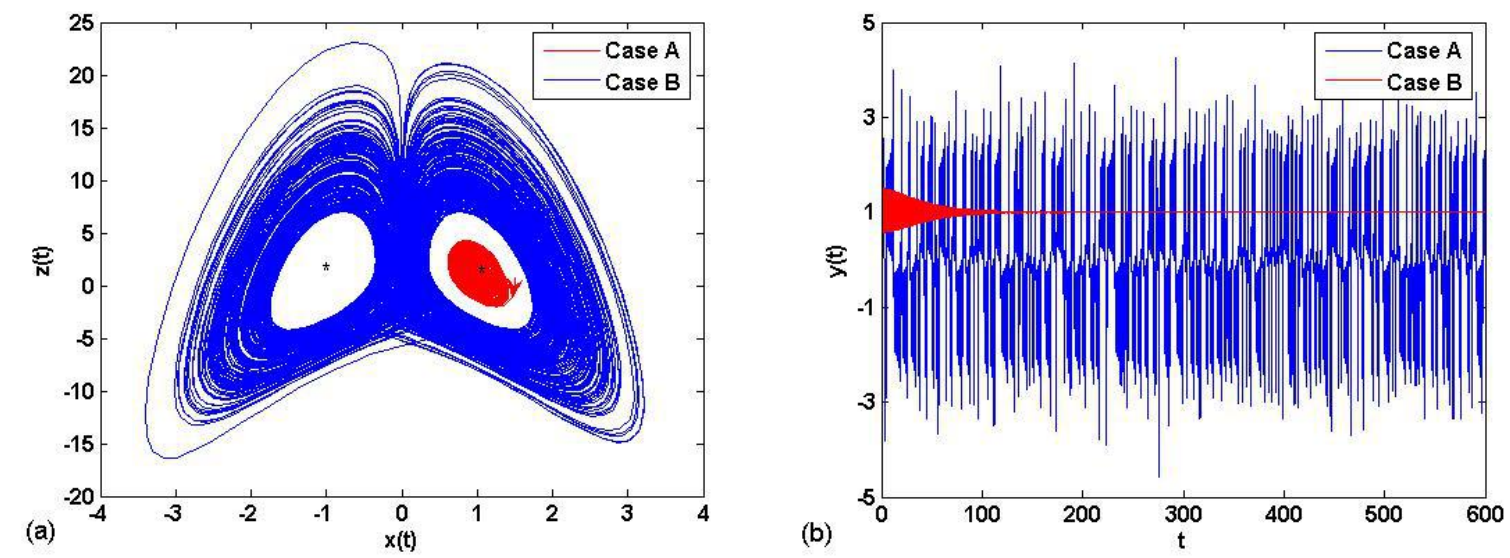

Figure 1. Phase portraits of system (1) with $r=10, m=0.75$ and $g=20$. Case A: for initial values $(2,2,0.75)$, Case $B$ : for initial values $(1.5,1.5,0.75)$. 


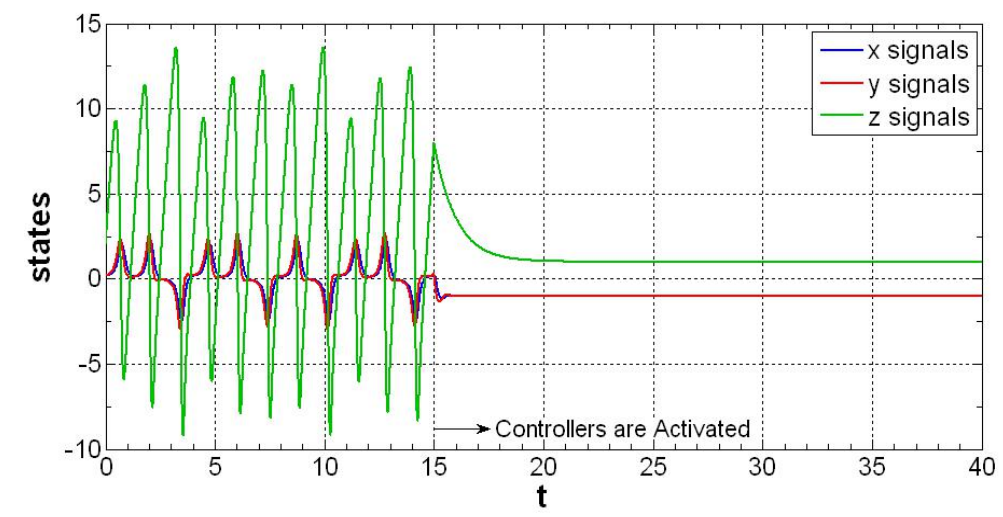

(a)

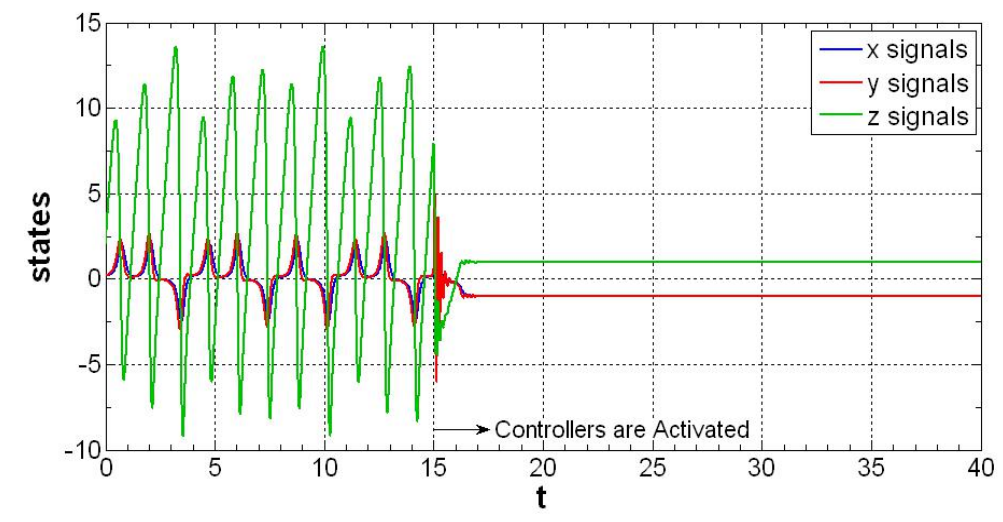

(c)

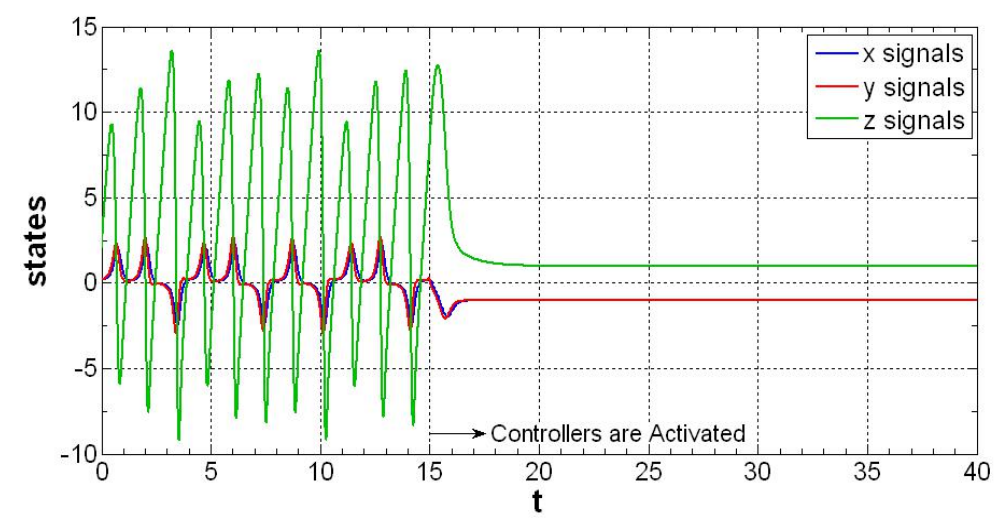

(b)

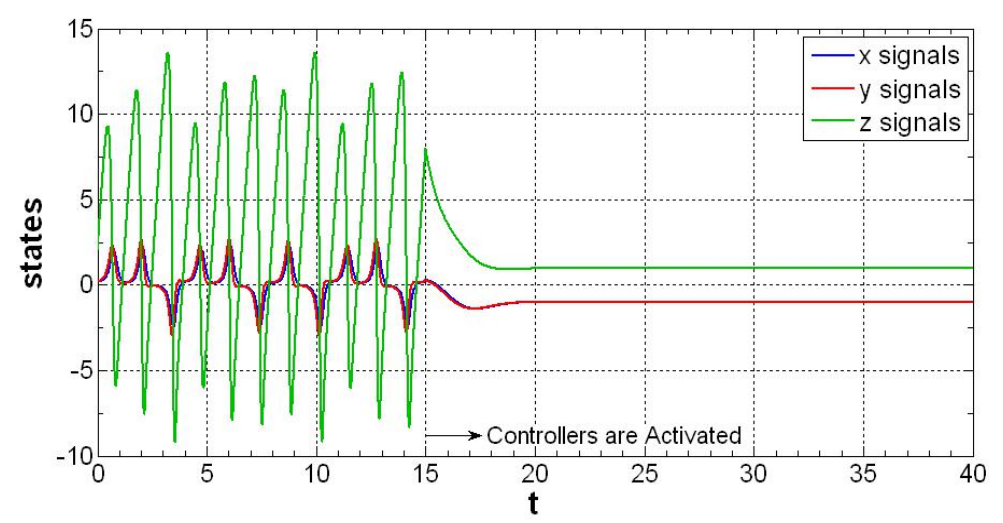

(d)

Figure 2. Time series of controlled self-exciting homopolar disc dynamo for $E_{1}(-1,-1,1)$ when the controllers are activated at $t=15$ with (a) Lyapunov based controllers, (b) sliding mode controllers, (c) hybrid controllers - case A, (d) hybrid controllers - case B. 


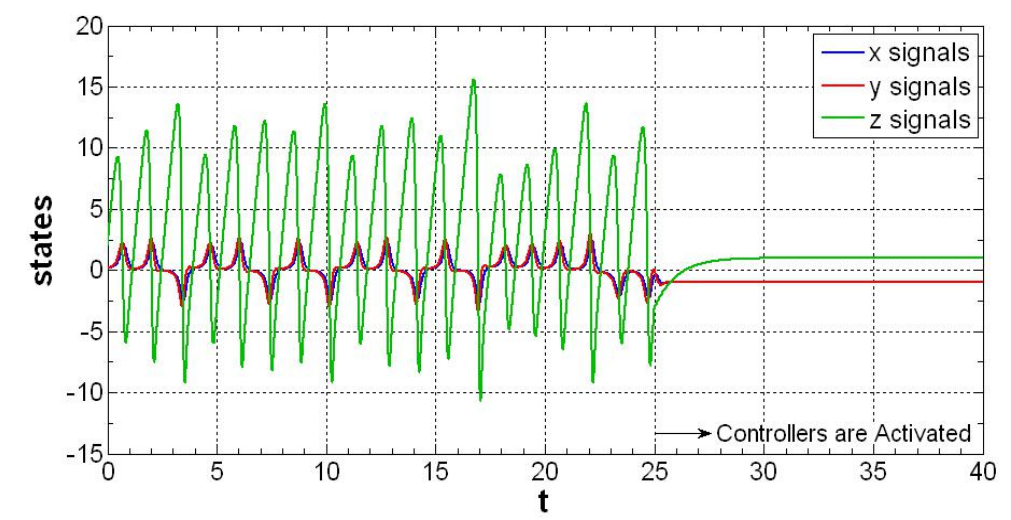

(a)

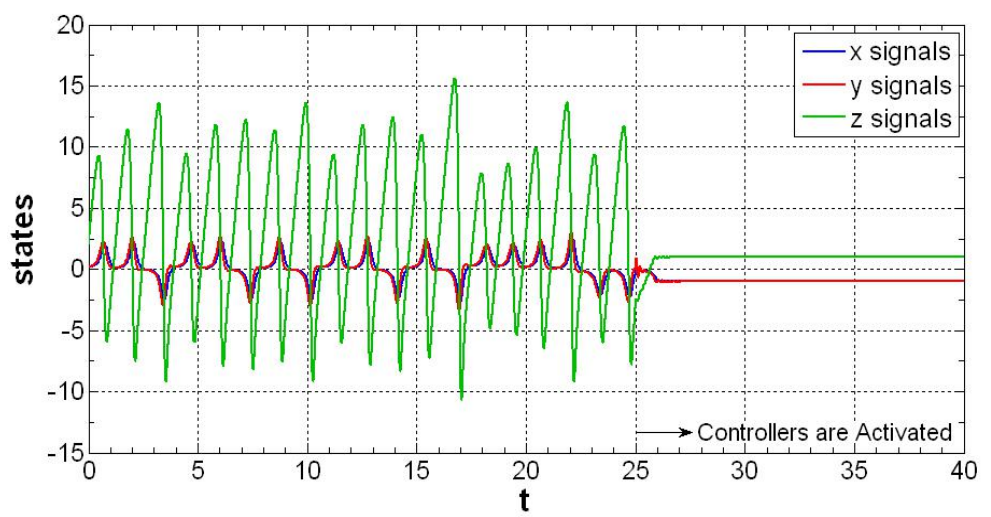

(c)

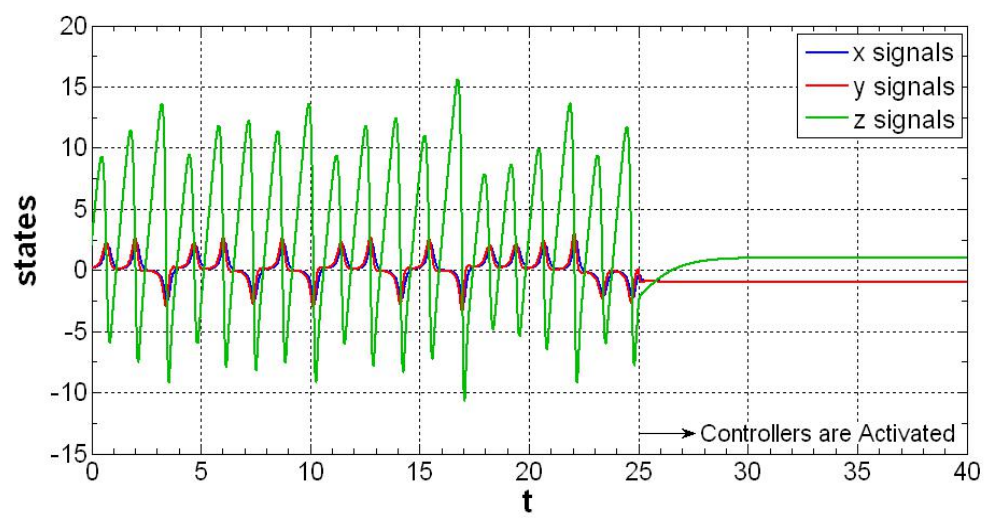

(b)

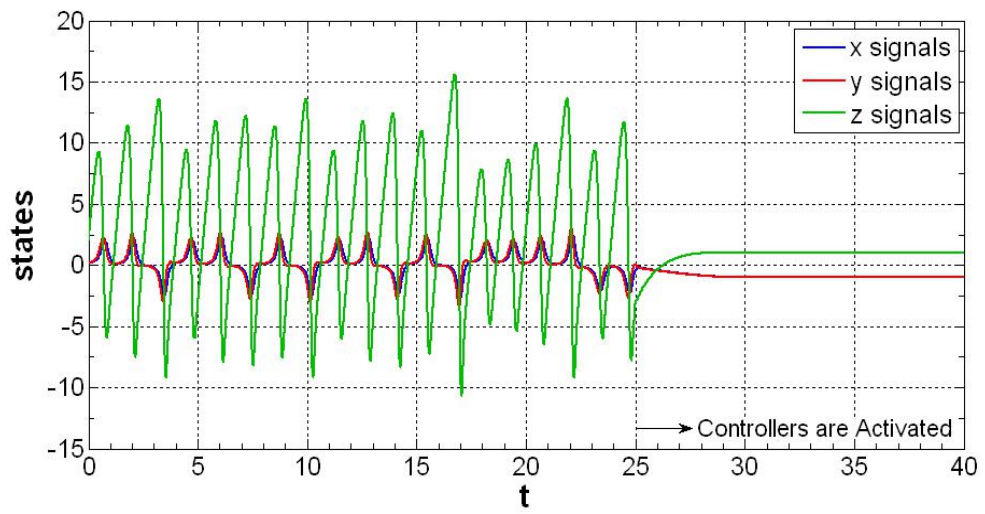

(d)

Figure 3. Time series of controlled self-exciting homopolar disc dynamo for $E_{1}(-1,-1,1)$ when the controllers are activated at $t=25$ with (a) Lyapunov based controllers, (b) sliding mode controllers, (c) hybrid controllers - case A, (d) hybrid controllers - case B. 


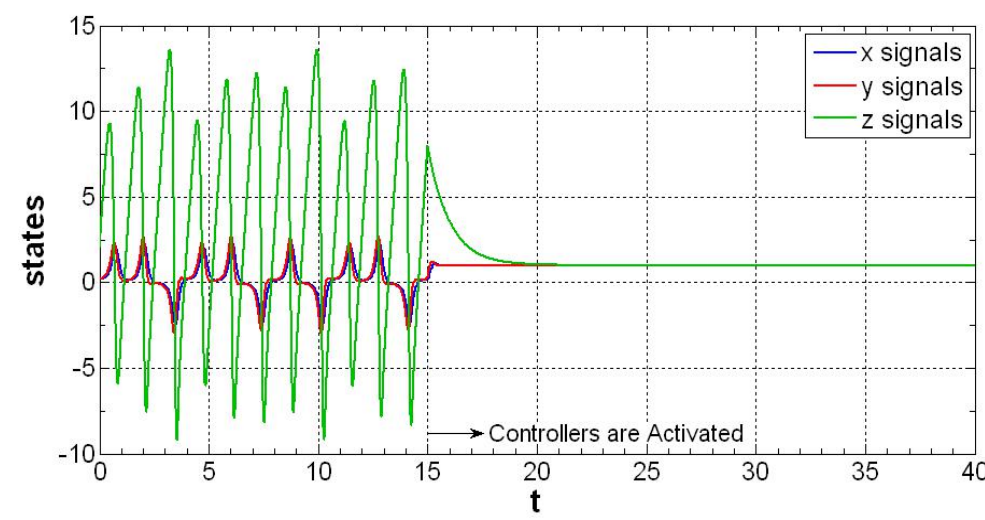

(a)

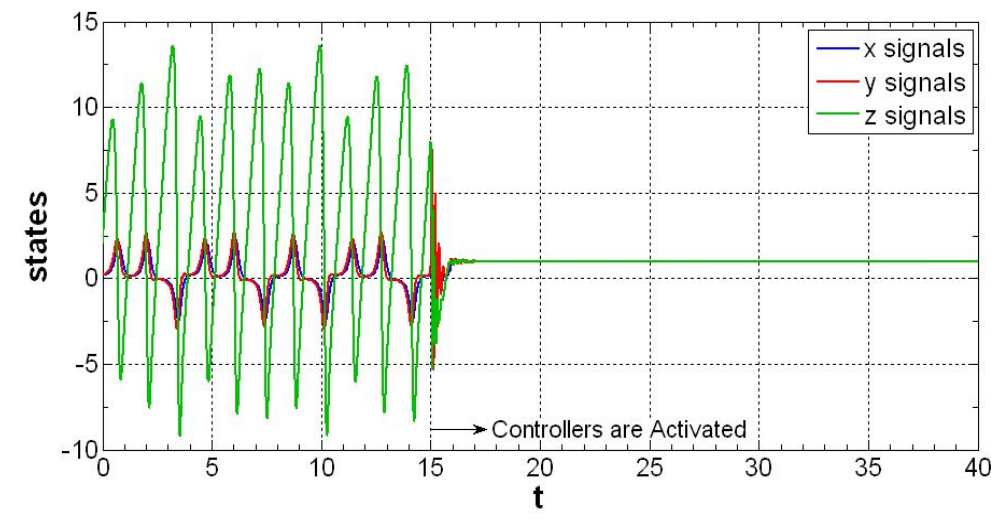

(c)

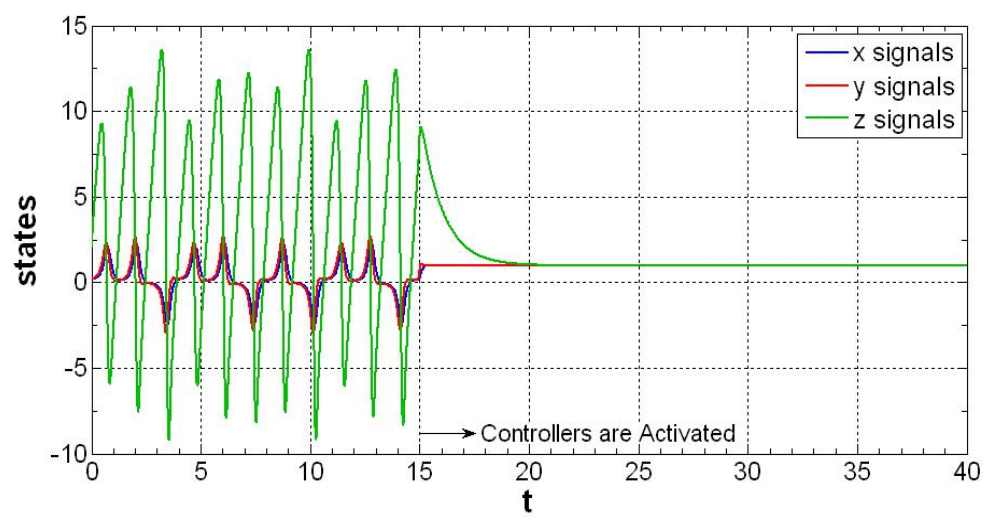

(b)

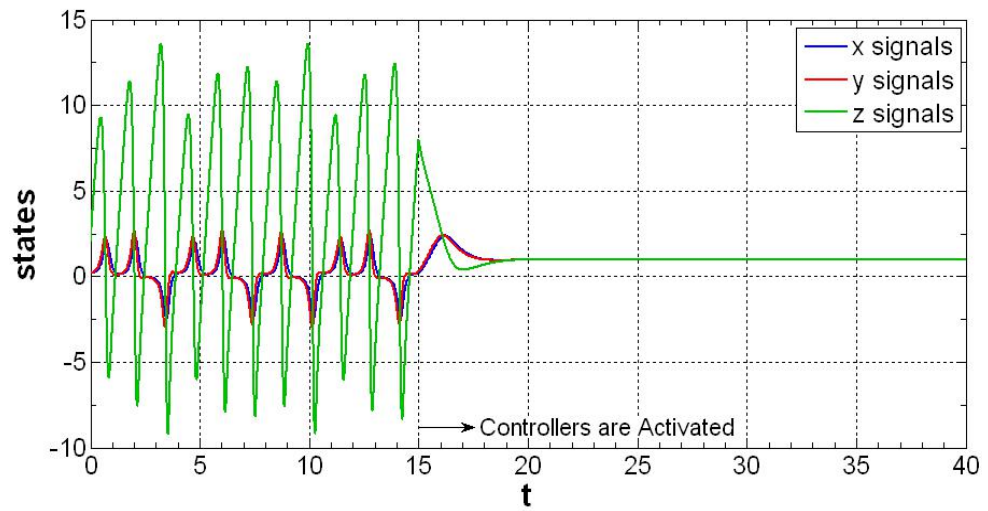

(d)

Figure 4. Time series of controlled self-exciting homopolar disc dynamo for $E_{2}(1,1,1)$ when the controllers are activated at $t=15$ with (a) Lyapunov based controllers, (b) sliding mode controllers, (c) hybrid controllers - case A, (d) hybrid controllers - case B. 


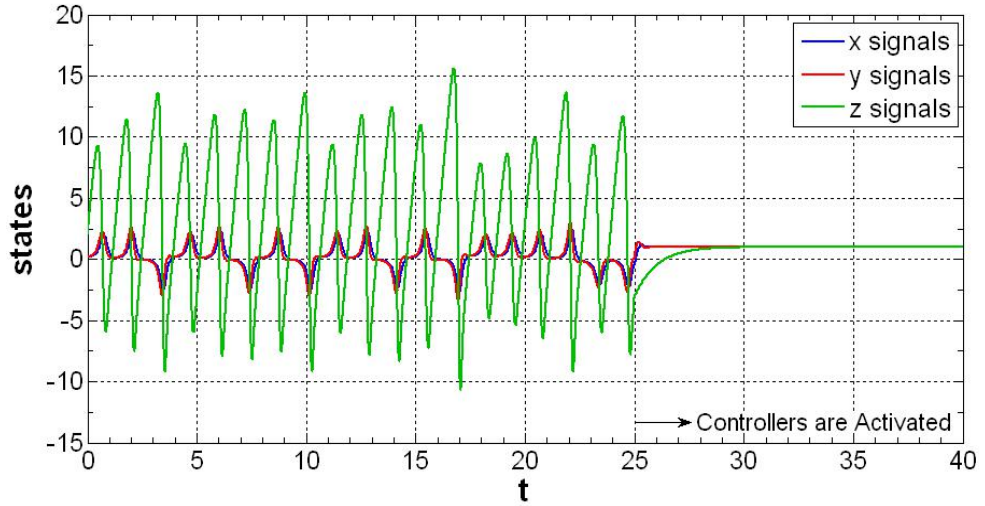

(a)

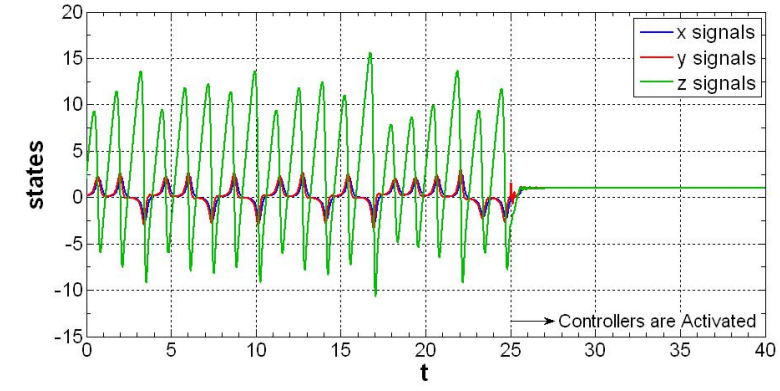

(c)

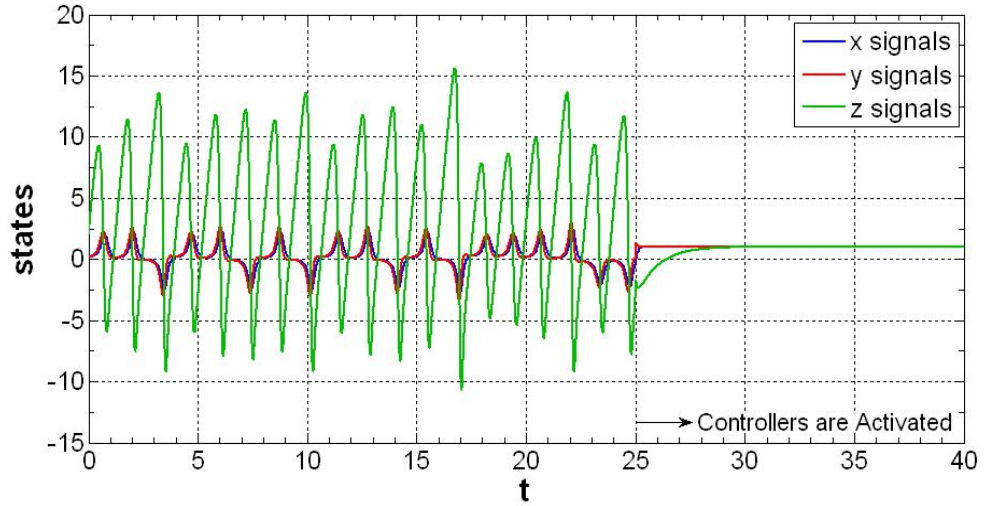

(b)

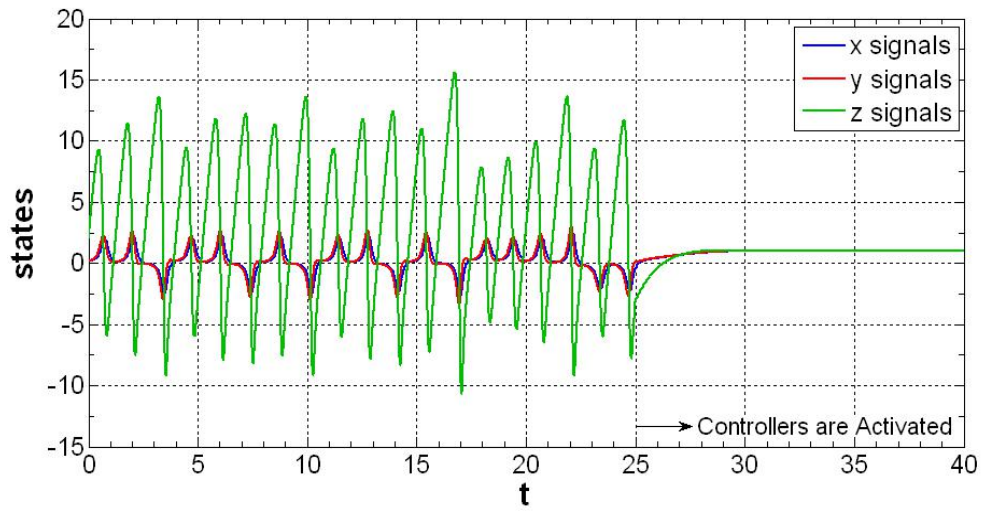

(d)

Figure 5. Time series of self-exciting homopolar disc dynamo for $E_{2}(1,1,1)$ when the controllers are activated at $t=25$ with (a) Lyapunov based controllers, (b) sliding mode controllers, (c) hybrid controllers - case A, (d) hybrid controllers - case B. 


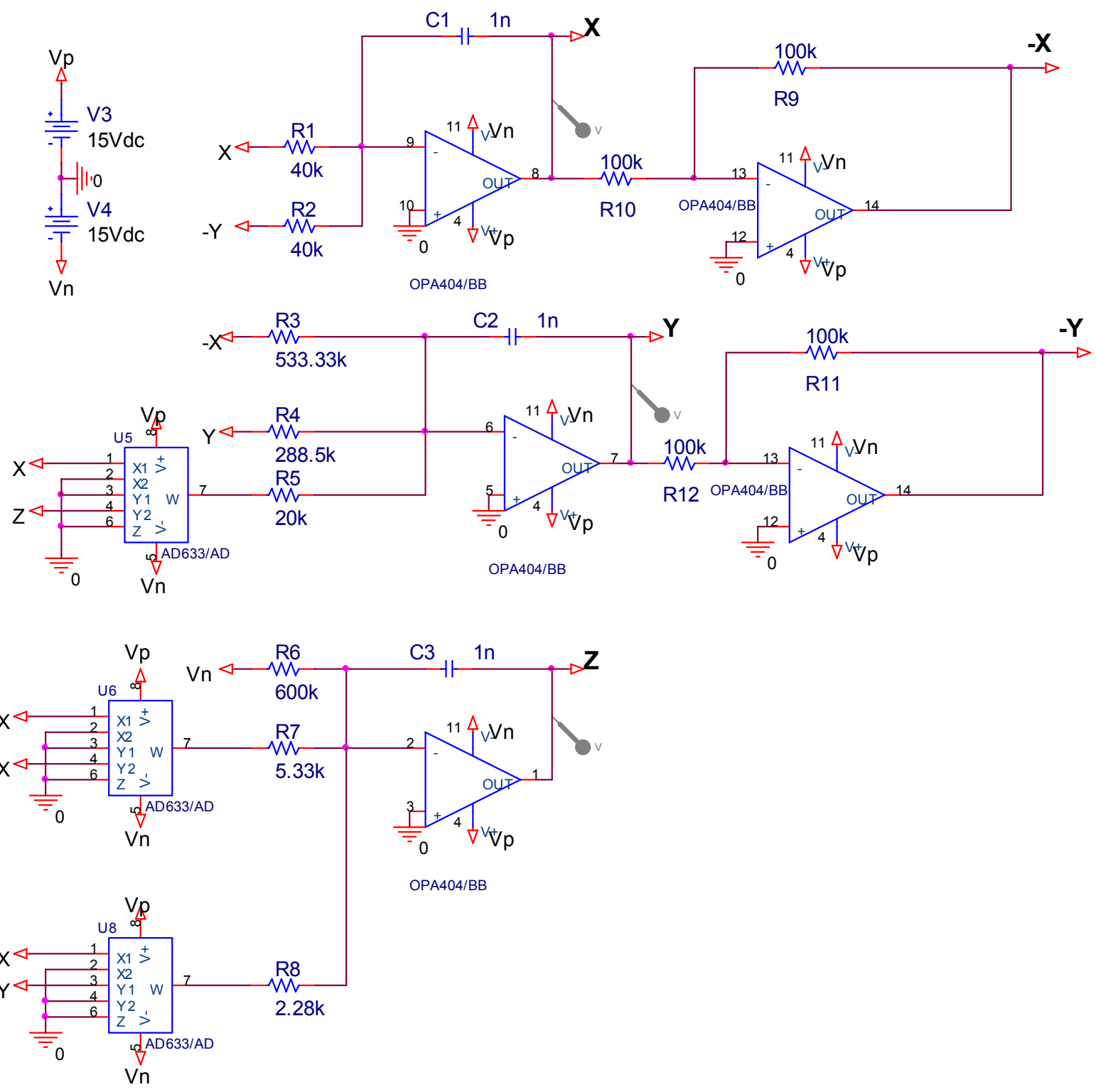

Figure 6. The electronic circuit schematic of the scaled hidden chaotic attractor. 


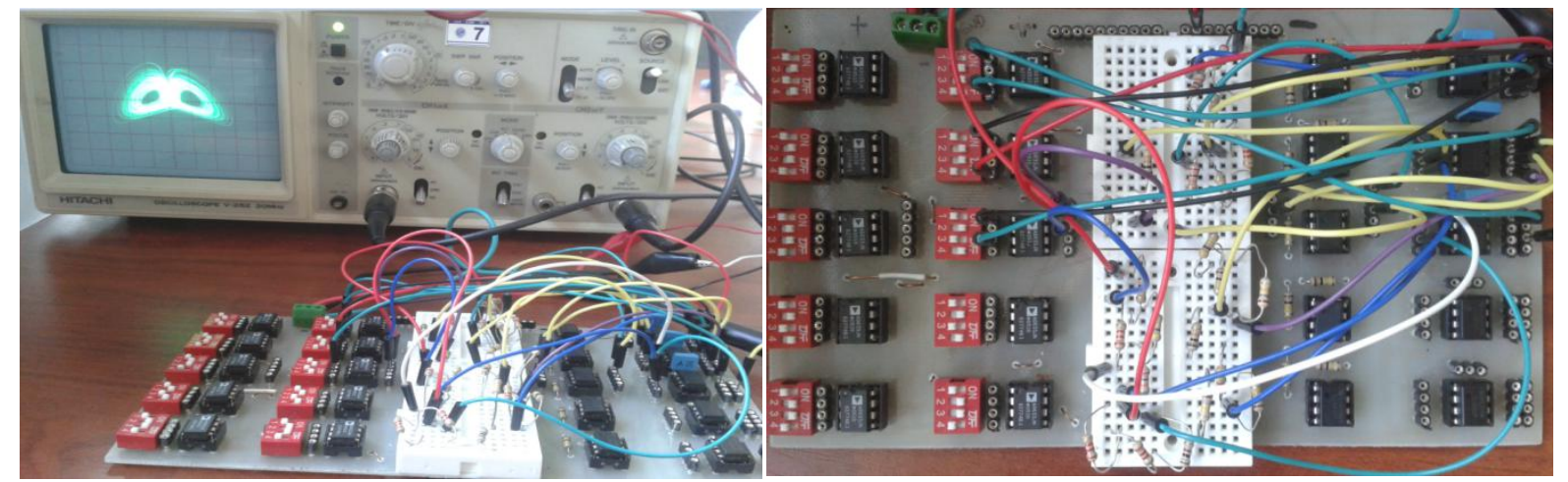

Figure 7. The experimental circuit of the hidden chaotic attractor.
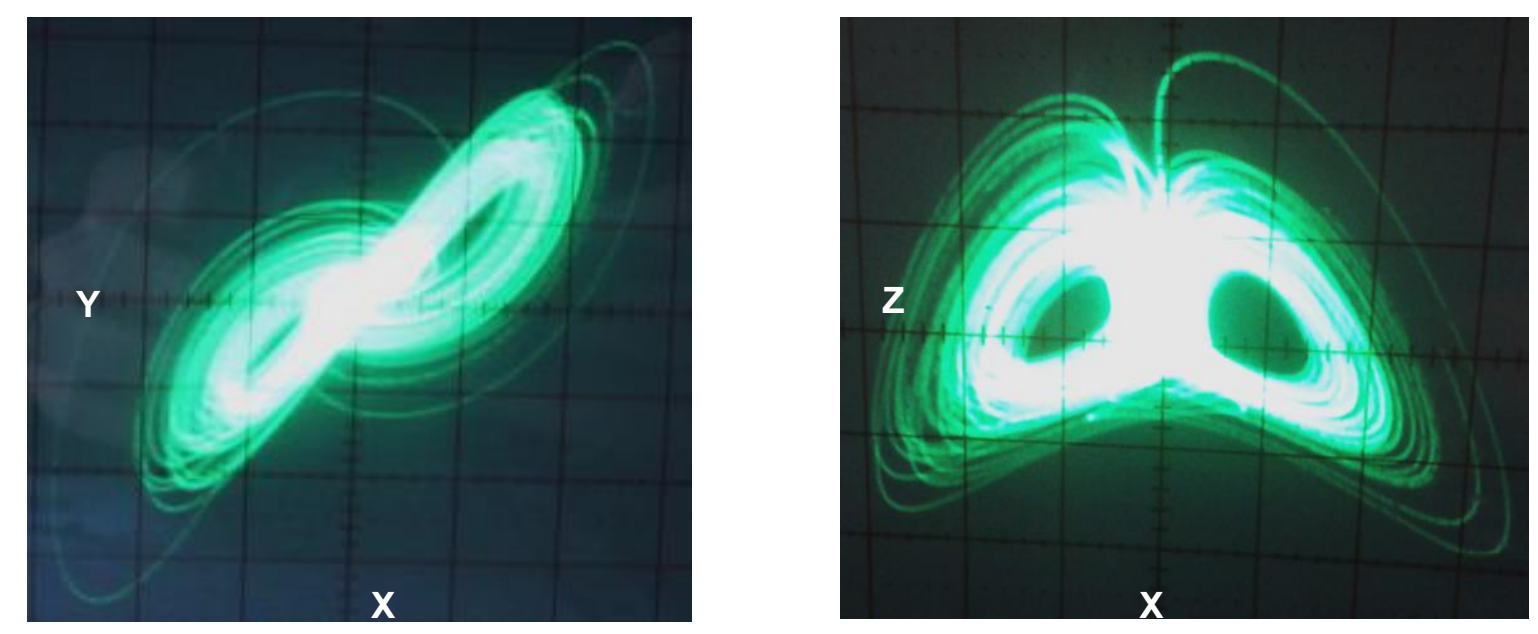

Figure 8. The phase portraits of scaled chaotic system with $r=10, m=0.75$ and $g=20$ on the oscilloscope outputs. 

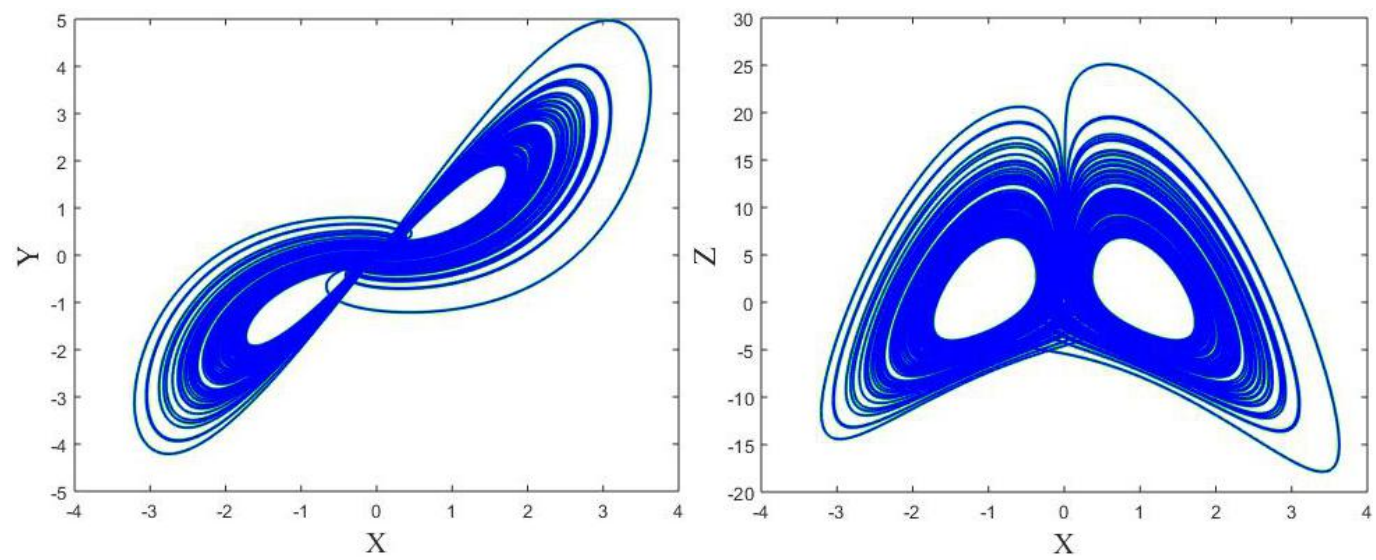

Figure 9. 2D phase portraits of FOCS system (43) for $r=10 ; m=0.75 ; g=20$ and commensurate fractional order $q=0.997$.

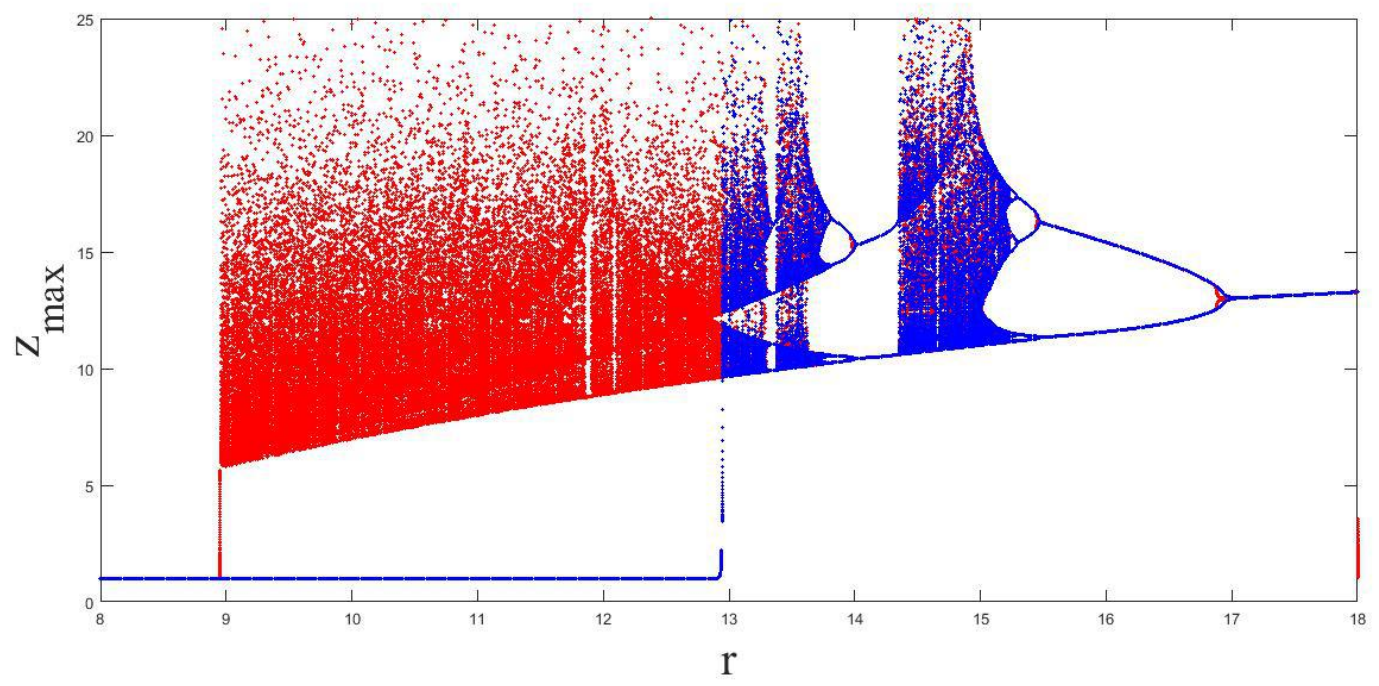

Figure 10. Bifurcation of the FOCS system (43) with parameter $r$. Red shows backward continuation and blue shows forward continuation . 


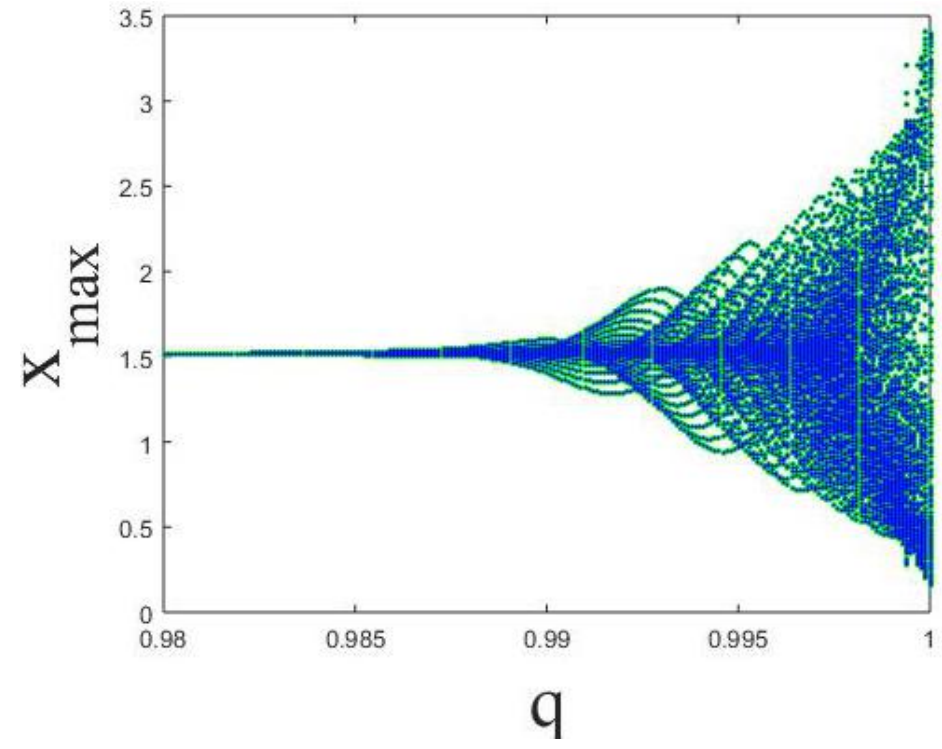

Figure 11. 2D phase portraits of FOCS system for fractional orders $q_{x}=q_{y}=q_{z}=q$.

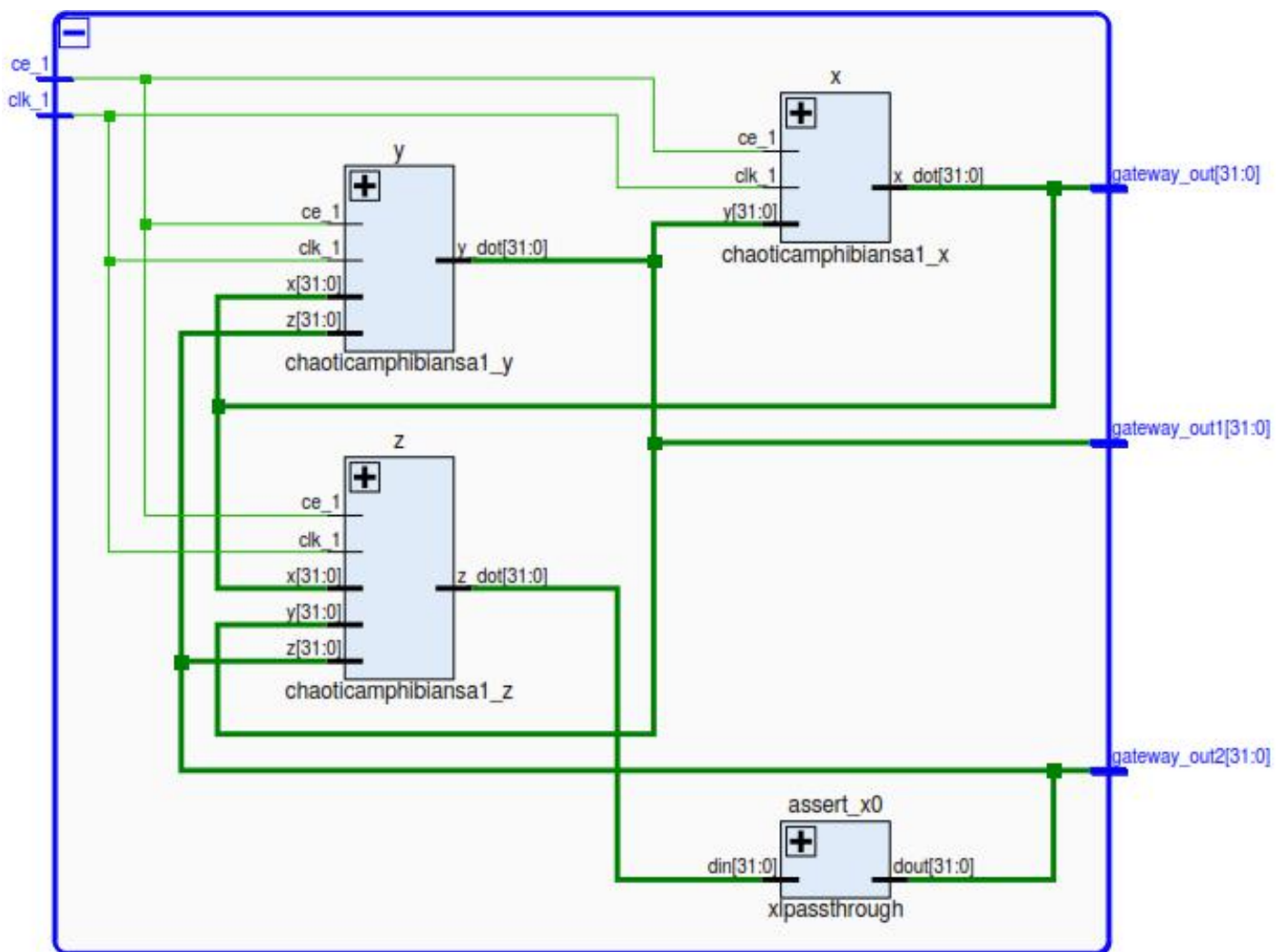

Figure 12. RTL schematics of FOCS system (43). 


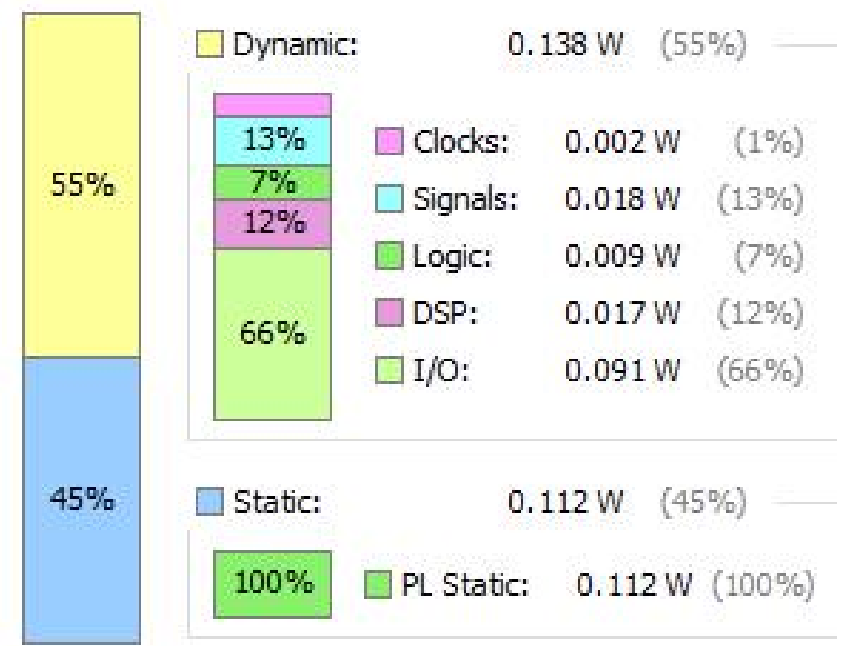

(a)

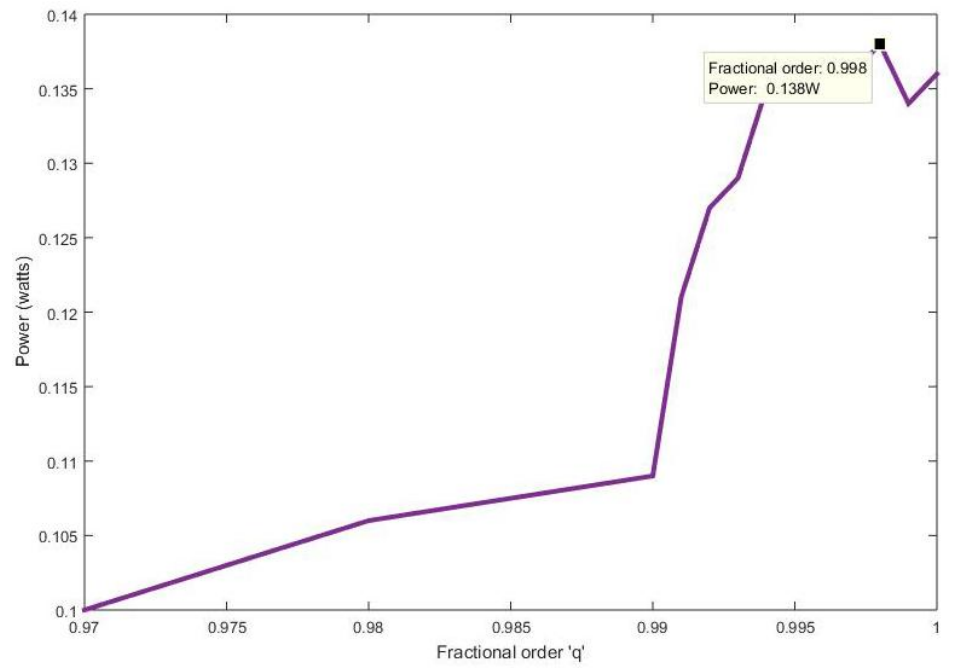

(b)

Figure 13. (a) Power utilized and (b) Power utilization vs fractional order of FOCS system (43).
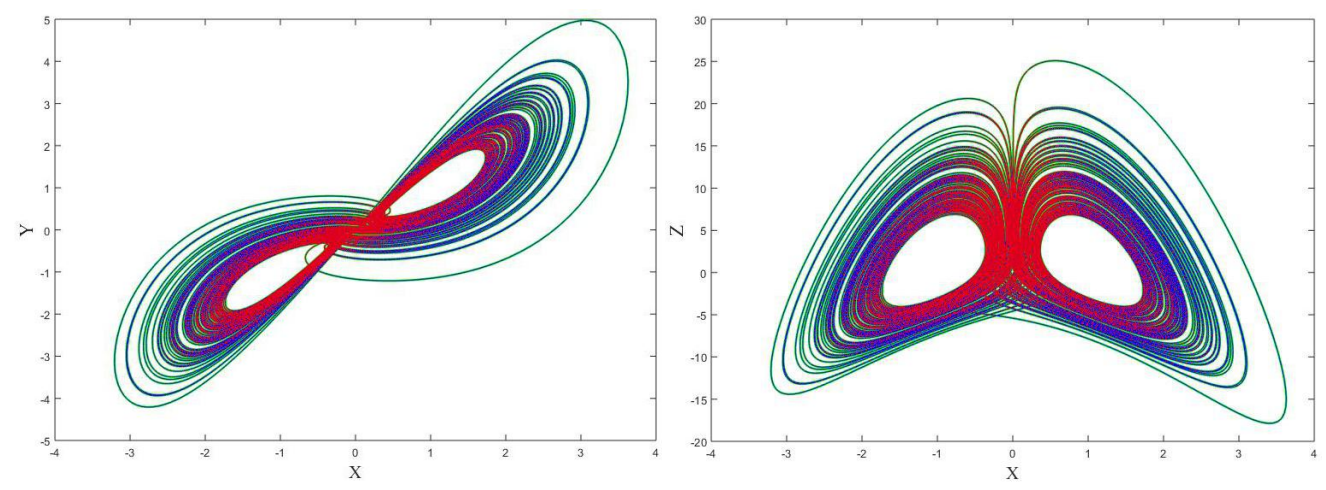

Figure 14. 2D phase portraits of the FPGA implemented FOCS for fractional order $q=0.997$. 


\begin{tabular}{|l|l|l|l|}
\hline Resource & Utilization & Available & $\begin{array}{c}\text { Utilization } \\
\mathbf{\%}\end{array}$ \\
\hline LUT & 725 & 101400 & 0.71 \\
\hline FF & 192 & 202800 & 0.09 \\
\hline DSP & 20 & 600 & 3.33 \\
\hline IO & 97 & 285 & 34.04 \\
\hline BUFG & 1 & 32 & 3.13 \\
\hline
\end{tabular}

Table I: Resource utilization of FOCS system (43). 